Supporting Information

\title{
Visible Light Absorption and Long-Lived Excited States in Dinuclear Silver(I) Complexes with Redox-Active Ligands
}

\author{
Dylan J. Shields, ${ }^{\ddagger}$ Tasneem Elkoush, ${ }^{\dagger}$ Emily Miura-Stempel, ${ }^{\dagger}$ \\ Choi L. Mak, ${ }^{\dagger}$ Guang-Hao Niu, ${ }^{\dagger}$ Anna D. Gudmundsdottir,,${ }^{\dagger}$ \\ and Michael G. Campbell ${ }^{*, \dagger}$ \\ ${ }^{\dagger}$ Department of Chemistry, Barnard College, New York, NY 10027, United States \\ ${ }^{\ddagger}$ Department of Chemistry, University of Cincinnati, Cincinnati, $\mathrm{OH} 45221$, United States \\ *E-mail: mcampbel@barnard.edu
}




\section{Table of Contents}

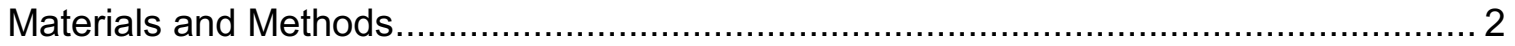

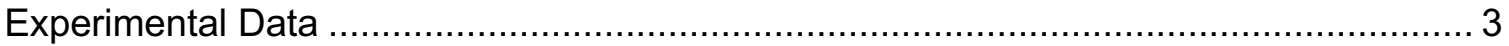

Synthesis and Characterization of New Compounds ............................................. 3

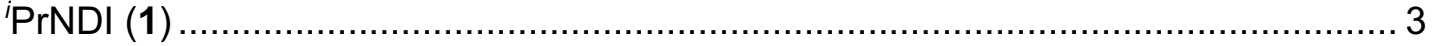

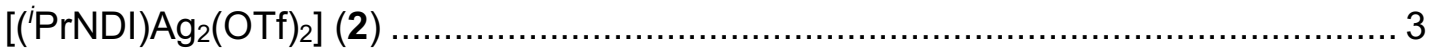

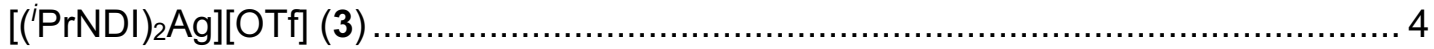

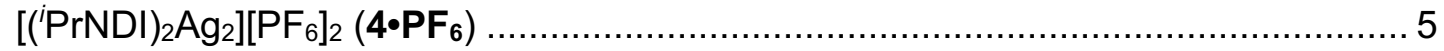

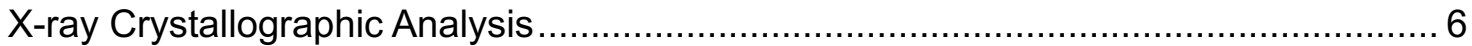

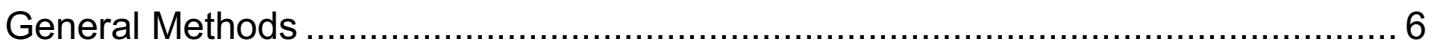

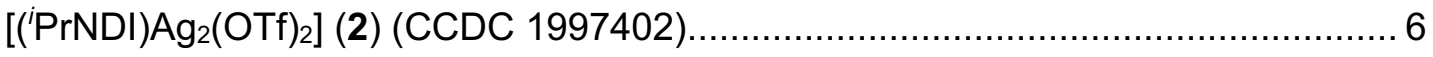

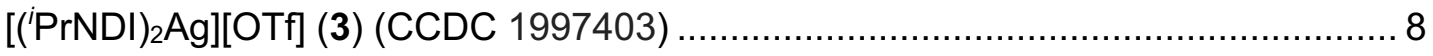

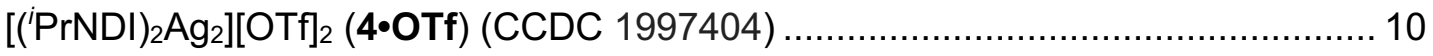

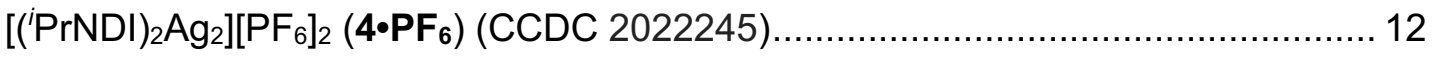

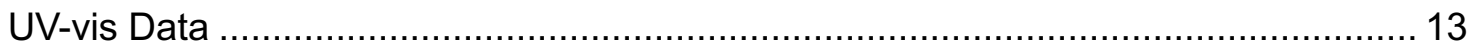

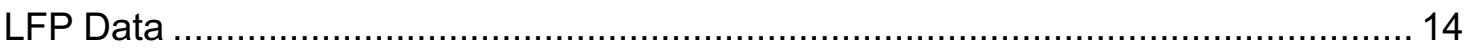

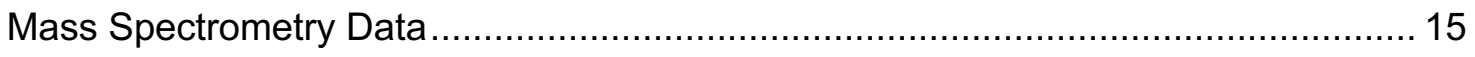

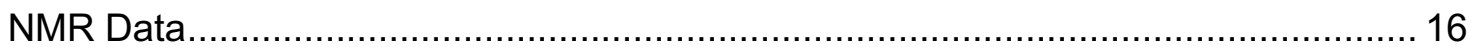

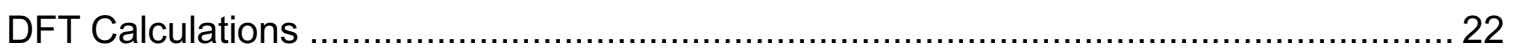

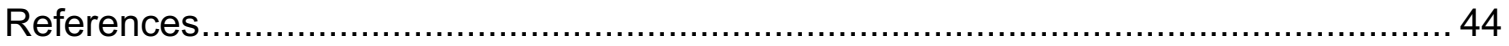




\section{Materials and Methods}

General Methods. All manipulations were carried out under an inert atmosphere $\left(\mathrm{N}_{2}\right.$ or $\mathrm{Ar}$ ) in a dry box or using conventional Schlenk techniques, unless otherwise noted. Anhydrous solvents were obtained by filtration through drying columns ${ }^{1}$ on an mBraun system, and were degassed by sparging with Ar or by three freeze-pump-thaw cycles before use. All solid reagents were dried overnight in a vacuum desiccator before being transferred into a dry box.

Reagents. Silver(I) trifluoromethanesulfonate and silver(I) hexafluorophosphate were purchased from Strem. 2,6-diisopropylaniline was purchased from Sigma Aldrich. 1,8naphthyridine-2,7-dicarboxaldehyde was synthesized according to the literature. ${ }^{2}$

Deuterated solvents were purchased from Cambridge Isotope Labs, dried over activated $4 \AA$ molecular sieves, and degassed by sparging with Ar prior to use.

Data Collection and Instrumentation. NMR spectra were recorded on a Bruker AVANCE spectrometer operating at $300 \mathrm{MHz}$ for ${ }^{1} \mathrm{H}$ and $75 \mathrm{MHz}$ for ${ }^{13} \mathrm{C}$ acquisitions, or a Bruker AVANCE NEO NanoBay spectrometer operating at $400 \mathrm{MHz}$ for ${ }^{1} \mathrm{H}, 100 \mathrm{MHz}$ for ${ }^{13} \mathrm{C}$, and $376 \mathrm{MHz}$ for ${ }^{19} \mathrm{~F}$ acquisitions. Chemical shifts are reported in ppm with the solvent resonance as the internal standard. The following solvent chemical shifts were used as reference values $(\mathrm{ppm}): \mathrm{CDCl}_{3}=7.26\left({ }^{1} \mathrm{H}\right), 77.16\left({ }^{13} \mathrm{C}\right) ; \mathrm{CD}_{2} \mathrm{Cl}_{2}=5.32\left({ }^{1} \mathrm{H}\right)$, $53.84\left({ }^{13} \mathrm{C}\right) ; \mathrm{CD}_{3} \mathrm{CN}=1.94\left({ }^{1} \mathrm{H}\right), 1.32,118.3\left({ }^{13} \mathrm{C}\right) .{ }^{3}$ Data are reported as follows:

multiplicity $(\mathrm{s}=$ singlet, $\mathrm{br}=$ broad, $\mathrm{d}=$ doublet, $\mathrm{t}=$ triplet, $\mathrm{q}=$ quartet, quin = quintet, hept $=$ heptet, $\mathrm{m}=$ multiplet); coupling constants in $\mathrm{Hz}$; integration. UV-vis spectra were collected on a Varian Cary 5000 spectrophotometer, using quartz cuvettes with a $1 \mathrm{~cm}$ path length. HRMS data were collected at the Columbia University Mass Spectrometry Core Facility, using a Waters XEVO G2-XS QToF mass spectrometer; for moisturesensitive compounds such as 2 , direct injection of anhydrous solutions was performed using a syringe pump. Laser flash photolysis measurements were performed using an Edinburgh detector, with $355 \mathrm{~nm}$ irradiation from a Continuum Nd:YAG laser; this system has been described in detail previously. ${ }^{4}$ Sample solutions were prepared using anhydrous $\mathrm{CH}_{2} \mathrm{Cl}_{2}$ in a dry box, and sealed in air-free quartz cuvettes. The same samples were used for fluorescence spectroscopy with $355 \mathrm{~nm}$ irradiation. For phosphorescence spectroscopy, $0.5 \mathrm{~mL}$ of a $\mathrm{CH}_{2} \mathrm{Cl}_{2}$ solution of $\mathbf{2}$ or $\mathbf{4} \cdot \mathrm{PF}_{6}$ was mixed with $0.5 \mathrm{~mL}$ of anhydrous toluene in a dry box. Glassy matrixes for phosphorescence studies were formed by submerging the samples in liquid $\mathrm{N}_{2}$ for at least 5 minutes prior to stimulation with $355 \mathrm{~nm}$ irradiation. 


\section{Experimental Data}

\section{Synthesis and Characterization of New Compounds}<smiles>O=Cc1ccc2ccc(C=O)nc2n1</smiles>

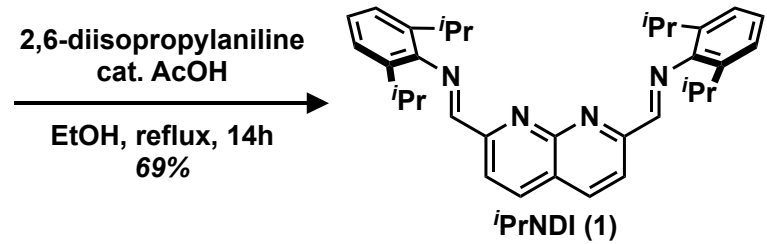

\section{'PrNDI (1)}

Under air, 1,8-naphthyridine-2,7-dicarboxaldehyde $(0.10 \mathrm{~g}, 0.54 \mathrm{mmol}, 1.0$ equiv. $)$ was suspended in EtOH (3 mL). 2,6-Diisopropylaniline $(0.22 \mathrm{~mL}, 1.1 \mathrm{mmol}, 2.1$ equiv.) was added by syringe. AcOH ( 2 drops) was added, and the reaction mixture was stirred at reflux for $14 \mathrm{~h}$. The reaction mixture was cooled to room temperature and then concentrated to dryness under vacuum. The residue was washed with cold EtOH several times, then dried under high vacuum to afford compound $\mathbf{1}$ as a pale yellow powder (0.19 g, $0.37 \mathrm{mmol}, 69 \%$ yield).

NMR spectroscopy: ${ }^{1} \mathrm{H}$ NMR $\left(300 \mathrm{MHz}, \mathrm{CDCl}_{3}, 23{ }^{\circ} \mathrm{C}, \delta\right): 8.64-8.51(\mathrm{~m}, 4 \mathrm{H}), 8.43(\mathrm{~d}, \mathrm{~J}=$ $8.4 \mathrm{~Hz}, 2 \mathrm{H}), 7.14-7.23(\mathrm{~m}, 6 \mathrm{H}), 3.01$ (hept, $J=6.8 \mathrm{~Hz}, 4 \mathrm{H}), 1.19(\mathrm{~d}, J=6.9 \mathrm{~Hz}, 24 \mathrm{H})$. ${ }^{13} \mathrm{C}$ NMR $\left(75 \mathrm{MHz}, \mathrm{CDCl}_{3}, 23^{\circ} \mathrm{C}, \delta\right): 163.0,157.9,155.7,147.9,137.8,137.1,124.9$, 123.2, 120.2, 28.0, 23.5.

Note: one expected aromatic carbon signal was not observed in the ${ }^{13} \mathrm{C}$ spectrum, even with longer acquisition times. This missing signal does not affect our assignment of the identity or purity of 1.

UV-vis spectroscopy $\left(\mathrm{CH}_{2} \mathrm{Cl}_{2}\right): 252 \mathrm{~nm}\left(\varepsilon=4.3 \times 10^{4} \mathrm{M}^{-1} \mathrm{~cm}^{-1}\right), 331 \mathrm{~nm}\left(\varepsilon=8.3 \times 10^{3}\right.$ $\left.\mathrm{M}^{-1} \mathrm{~cm}^{-1}\right), 345 \mathrm{~nm}\left(\varepsilon=9.2 \times 10^{3} \mathrm{M}^{-1} \mathrm{~cm}^{-1}\right), \sim 350-450 \mathrm{~nm}$ (broad shoulder).

HRMS (ESI+) m/z: [M+H] $]^{+}$calcd for $\mathrm{C}_{34} \mathrm{H}_{41} \mathrm{~N}_{4}, 505.3331$; found, 505.3337.

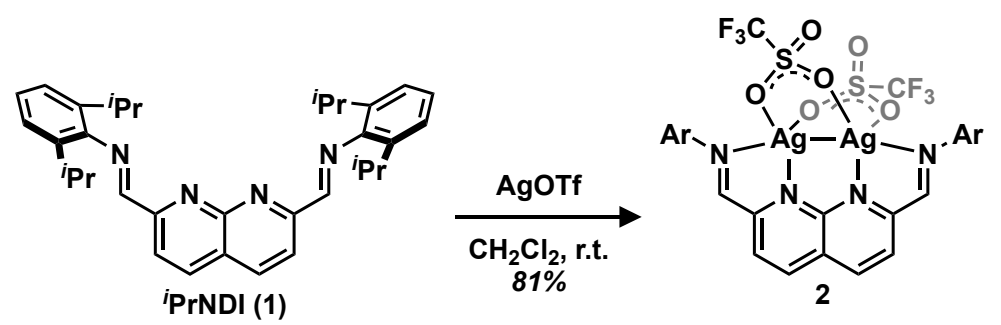

\section{[('PrNDI)Ag $\left.{ }_{2}(\mathrm{OTf})_{2}\right](2)$}

'PrNDI (1) (0.020 g, 0.040 mmol, 1.0 equiv) was dissolved in $\mathrm{CH}_{2} \mathrm{Cl}_{2}(1.5 \mathrm{~mL}$ ), affording a clear yellow solution. The solution of 1 was then transferred to a suspension of silver triflate $\left(0.020 \mathrm{~g}, 0.079 \mathrm{mmol}, 2.0\right.$ equiv) in $\mathrm{CH}_{2} \mathrm{Cl}_{2}(1.5 \mathrm{~mL})$, and the mixture was stirred vigorously at room temperature. An orange color developed within the first 5 minutes of stirring, followed by further development of a red color after stirring overnight. The red solution was filtered to remove any residual solids, and then divided into two $4 \mathrm{~mL}$ vials. These vials were each placed, uncapped, into $20 \mathrm{~mL}$ vials containing approximately 4 
$\mathrm{mL}$ of $\mathrm{Et}_{2} \mathrm{O}$, and then the $20 \mathrm{~mL}$ vials were capped and stored at room temperature. After sitting overnight, vapor diffusion of $\mathrm{Et}_{2} \mathrm{O}$ into the $\mathrm{CH}_{2} \mathrm{Cl}_{2}$ solution resulted in the formation of orange-red crystals. The mother liquor was carefully decanted, and the solids were isolated and dried under high vacuum, affording complex 2 as a red crystalline powder (33 $\mathrm{mg}, 0.032 \mathrm{mmol}, 81 \%$ yield).

NMR spectroscopy: ${ }^{1} \mathrm{H}$ NMR (400 MHz, $\left.\mathrm{CD}_{2} \mathrm{Cl}_{2}, 25^{\circ} \mathrm{C}, \delta\right): 8.96(\mathrm{~d}, \mathrm{~J}=8.3 \mathrm{~Hz}, 2 \mathrm{H}), 8.66$ (s, 2H), $8.18(\mathrm{~d}, J=8.3 \mathrm{~Hz}, 2 \mathrm{H}), 7.47-7.16(\mathrm{~m}, 6 \mathrm{H}), 3.09$ (hept, $J=6.8 \mathrm{~Hz}, 4 \mathrm{H}), 1.24$ (d, $J=6.8 \mathrm{~Hz}, 24 \mathrm{H}) .{ }^{13} \mathrm{C}$ NMR $\left(100 \mathrm{MHz}, \mathrm{CD}_{2} \mathrm{Cl}_{2}, 25{ }^{\circ} \mathrm{C}, \delta\right): 161.9,153.5,151.1,145.8$, 142.4, 138.6, 128.7, 127.7, 127.0, $124.6\left(\underline{\mathrm{CF}}_{3} \mathrm{SO}_{3}{ }^{-}\right), 123.7,121.8\left(\underline{\mathrm{CF}}_{3} \mathrm{SO}_{3}{ }^{-}\right), 118.7$ $\left(\mathrm{CF}_{3} \mathrm{SO}_{3}{ }^{-}\right), 28.2,23.5(\mathrm{br}) .{ }^{19} \mathrm{~F} \mathrm{NMR}\left(376 \mathrm{MHz}, \mathrm{CD}_{2} \mathrm{Cl}_{2}, 25{ }^{\circ} \mathrm{C}, \delta\right):-77.3$.

UV-vis spectroscopy $\left(\mathrm{CH}_{2} \mathrm{Cl}_{2}\right): 257 \mathrm{~nm}\left(\varepsilon=2.3 \times 10^{4} \mathrm{M}^{-1} \mathrm{~cm}^{-1}\right), 351 \mathrm{~nm}\left(\varepsilon=9.7 \times 10^{3}\right.$ $\left.\mathrm{M}^{-1} \mathrm{~cm}^{-1}\right), 364 \mathrm{~nm}\left(\varepsilon=9.5 \times 10^{3} \mathrm{M}^{-1} \mathrm{~cm}^{-1}\right), 467 \mathrm{~nm}\left(\varepsilon=1.8 \times 10^{3} \mathrm{M}^{-1} \mathrm{~cm}^{-1}\right)$.

Details of SCXRD data collection and analysis are reported in the X-ray Crystallographic Analysis section below. HRMS data is shown in Figure S8.

If the reaction mixture is stirred just until full dissolution of the AgOTf, diffusion of $\mathrm{Et}_{2} \mathrm{O}$ into the resulting orange solution instead yields yellow crystals of the mononuclear complex [('PrNDI) $\left.{ }_{2} \mathrm{Ag}\right][\mathrm{OTf}]$ (3). Independent synthesis and characterization of complex 3 is described below.

If complex 2 is exposed to ambient atmosphere, trace amounts of water, or Lewis basic solvents such as MeCN, ligand scrambling affords $\left[\left({ }^{\mathrm{P} P N D I}\right)_{2} \mathrm{Ag}_{2}\right][\mathrm{OTf}]_{2}(\mathbf{4} \bullet \mathrm{OTf}) .{ }^{1} \mathrm{H} N M R$ data indicates that in solutions that are not rigorously dry, 2 and $\mathbf{4}$ are in equilibrium (Figure S22). Crystallization from either wet $\mathrm{CH}_{2} \mathrm{Cl}_{2}$ solutions or MeCN solutions affords 4-OTf. Independent synthesis and characterization of complex 4 with non-coordinating hexafluorophosphate anions $\left(\mathbf{4} \cdot \mathbf{P F}_{6}\right)$ is described below.

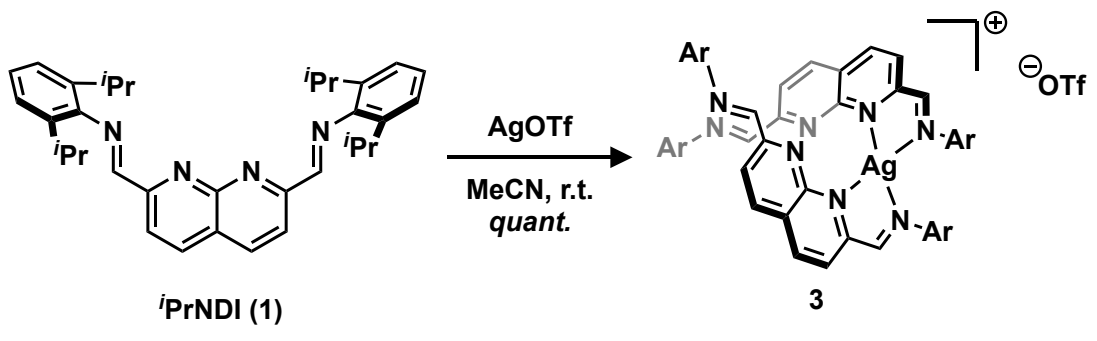

\section{[('PrNDI) ${ }_{2}$ Ag] [OTf] (3)}

'PrNDI (1) (0.02 g, $0.04 \mathrm{mmol}, 2$ equiv) and silver triflate (0.005 g, $0.02 \mathrm{mmol}, 1$ equiv) were combined in $\mathrm{MeCN}(2 \mathrm{~mL})$, and the mixture was stirred vigorously at room temperature. After 12 hours, the homogeneous pale orange solution was concentrated to dryness under vacuum, affording $0.025 \mathrm{~g}$ of complex 3 (quant. yield).

NMR spectroscopy: ${ }^{1} \mathrm{H}$ NMR $\left(400 \mathrm{MHz}, \mathrm{CD}_{3} \mathrm{CN}, 25^{\circ} \mathrm{C}, \delta\right): 8.75$ (d, J=8.4 Hz, 2H), 8.44 $-8.26(\mathrm{~m}, 4 \mathrm{H}), 7.18-7.16(\mathrm{~m}, 6 \mathrm{H}), 2.94$ (hept, $J=6.8 \mathrm{~Hz}, 4 \mathrm{H}), 0.92(\mathrm{~d}, J=6.9 \mathrm{~Hz}$, $24 \mathrm{H}) .{ }^{13} \mathrm{C}$ NMR $\left(100 \mathrm{MHz}, \mathrm{CD}_{3} \mathrm{CN}, 25^{\circ} \mathrm{C}, \delta\right): 163.6,156.3,154.2,148.2,141.1,138.3$, 127.6, 126.7, 124.5, 124.4, 28.8, 23.5. $\left.{ }^{19} \mathrm{~F} \mathrm{NMR} \mathrm{(376} \mathrm{MHz,} \mathrm{CD}_{3} \mathrm{CN}, 25{ }^{\circ} \mathrm{C}, \delta\right):-79.3$.

UV-vis spectroscopy $\left(\mathrm{CH}_{3} \mathrm{CN}\right): 251 \mathrm{~nm}\left(\varepsilon=1.0 \times 10^{5} \mathrm{M}^{-1} \mathrm{~cm}^{-1}\right), 332 \mathrm{~nm}\left(\varepsilon=1.9 \times 10^{4}\right.$ 
$\left.\mathrm{M}^{-1} \mathrm{~cm}^{-1}\right), 345 \mathrm{~nm}\left(\varepsilon=2.1 \times 10^{4} \mathrm{M}^{-1} \mathrm{~cm}^{-1}\right), \sim 360-450 \mathrm{~nm}$ (broad shoulder).

$X$-ray quality crystals were obtained via diffusion of $\mathrm{Et}_{2} \mathrm{O}$ into a saturated $\mathrm{CH}_{2} \mathrm{Cl}_{2}$ solution of 3 at $-35^{\circ} \mathrm{C}$. Details of SCXRD data collection and analysis are reported in the X-ray Crystallographic Analysis section below. UV-vis data is shown in Figure S5. HRMS data is shown in Figure S9.

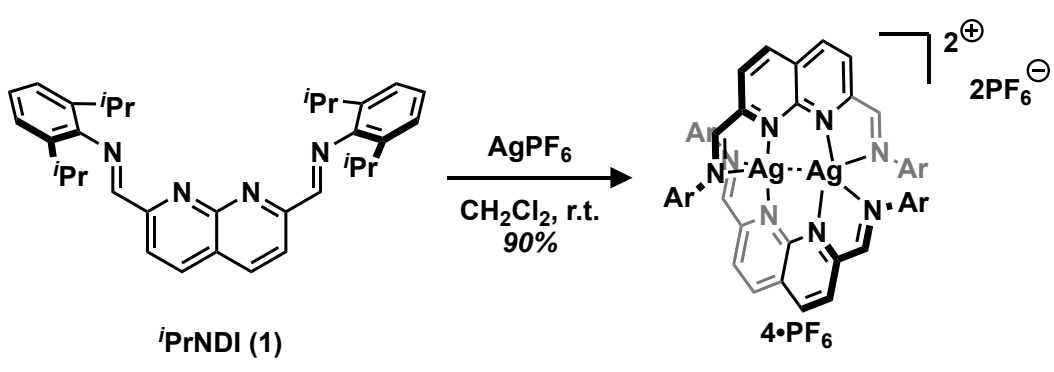

\section{$\left[\left({ }^{\prime} \mathrm{PrNDI}\right)_{2} \mathrm{Ag}_{2}\right]\left[\mathrm{PF}_{6}\right]_{2}\left(\mathbf{4} \cdot \mathrm{PF}_{6}\right)$}

'PrNDI (1) (0.020 g, $0.040 \mathrm{mmol}, 1.0$ equiv) was dissolved in $\mathrm{CH}_{2} \mathrm{Cl}_{2}(3 \mathrm{~mL})$, affording a clear yellow solution. The solution of 1 was then transferred to a vial containing silver hexafluorophosphate $(0.010 \mathrm{~g}, 0.040 \mathrm{mmol}, 1.0$ equiv $)$, affording a cloudy red/orange suspension. After stirring overnight at room temperature, the reaction mixture was a homogeneous cherry red solution. The solution was filtered to remove any residual solids, and then divided into three $4 \mathrm{~mL}$ vials. These vials were each placed, uncapped, into $20 \mathrm{~mL}$ vials containing approximately $4 \mathrm{~mL}$ of $\mathrm{Et}_{2} \mathrm{O}$, and then the $20 \mathrm{~mL}$ vials were capped and stored at room temperature. After sitting overnight, vapor diffusion of $\mathrm{Et}_{2} \mathrm{O}$ into the $\mathrm{CH}_{2} \mathrm{Cl}_{2}$ solution resulted in the formation of orange-red crystals. The mother liquor was carefully decanted, and the solids were isolated and dried under high vacuum, affording complex $4 \cdot \mathbf{P F}_{6}$ as a red crystalline powder $(27 \mathrm{mg}, 0.036 \mathrm{mmol}, 90 \%$ yield).

NMR spectroscopy: ${ }^{1} \mathrm{H}$ NMR (400 MHz, $\left.\mathrm{CD}_{2} \mathrm{Cl}_{2}, 25^{\circ} \mathrm{C}, \delta\right): 9.35$ (d, J = $\left.8.2 \mathrm{~Hz}, 2 \mathrm{H}\right), 8.77$ (s, 2H), $8.43(\mathrm{~d}, J=8.3 \mathrm{~Hz}, 2 \mathrm{H}), 7.26-7.14(\mathrm{~m}, 2 \mathrm{H}), 7.08(\mathrm{~d}, J=7.8 \mathrm{~Hz}, 4 \mathrm{H}), 2.77$ (hept, $J=6.8 \mathrm{~Hz}, 4 \mathrm{H}), 0.64(\mathrm{~d}, J=6.8 \mathrm{~Hz}, 24 \mathrm{H}) .{ }^{13} \mathrm{C}$ NMR $\left(100 \mathrm{MHz}, \mathrm{CD}_{2} \mathrm{Cl}_{2}, 25^{\circ} \mathrm{C}, \delta\right)$ : $161.2,155.3,152.6,144.5,144.3,138.3,128.7,127.7,127.0,124.3,28.9,23.0 .{ }^{19} \mathrm{~F}$ $\operatorname{NMR}\left(376 \mathrm{MHz}, \mathrm{CD}_{2} \mathrm{Cl}_{2}, 25^{\circ} \mathrm{C}, \delta\right):-72.6\left(\mathrm{~d},{ }^{1} \mathrm{JPF}_{\mathrm{PF}}=711 \mathrm{~Hz}\right)$.

UV-vis spectroscopy $\left(\mathrm{CH}_{2} \mathrm{Cl}_{2}\right): 254 \mathrm{~nm}\left(\varepsilon=4.7 \times 10^{4} \mathrm{M}^{-1} \mathrm{~cm}^{-1}\right), 348 \mathrm{~nm}\left(\varepsilon=1.9 \times 10^{4}\right.$ $\left.\mathrm{M}^{-1} \mathrm{~cm}^{-1}\right), 460 \mathrm{~nm}\left(\varepsilon=4.8 \times 10^{3} \mathrm{M}^{-1} \mathrm{~cm}^{-1}\right)$.

Details of SCXRD data collection and analysis are reported in the X-ray Crystallographic Analysis section below. HRMS data is shown in Figure S10.

If a 1:2 ratio of $1: A g P F_{6}$ is used, $1: 1$ complex $\mathbf{4} \cdot \mathbf{P F}_{6}$ is still obtained as the major product after crystallization, rather than a 1:2 complex analogous to 2. 


\section{X-ray Crystallographic Analysis}

\section{General Methods}

A single crystal was mounted on a polymer loop using Paratone- $\mathrm{N}$ oil, and transferred to an Agilent SuperNova diffractometer using mirror-monochromated Mo K $\alpha$ or $\mathrm{Cu} \mathrm{K} \alpha$ radiation. The sample was held at $100 \mathrm{~K}$ during the experiment. Data collection, integration, scaling (ABSPACK) and absorption correction were performed in CrysAlisPro. ${ }^{5}$ Structure solution was performed using ShelXT ${ }^{6}$ and subsequent refinement was performed by full-matrix least-squares on $\mathrm{F}^{2}$ in ShelXL, using the Olex2 interface. ${ }^{7}$ Non-hydrogen atoms were refined anisotropically, and hydrogen atoms were allowed to ride on their respective atoms. Graphics were prepared using CrystalMaker. ${ }^{8}$ Crystal details, as well as parameters for data collection and refinement, are given in the tables below.

\section{$\left[\left({ }^{\prime} P r N D I\right) A_{2}(\mathrm{OTf})_{2}\right](2)(C C D C$ 1997402)}

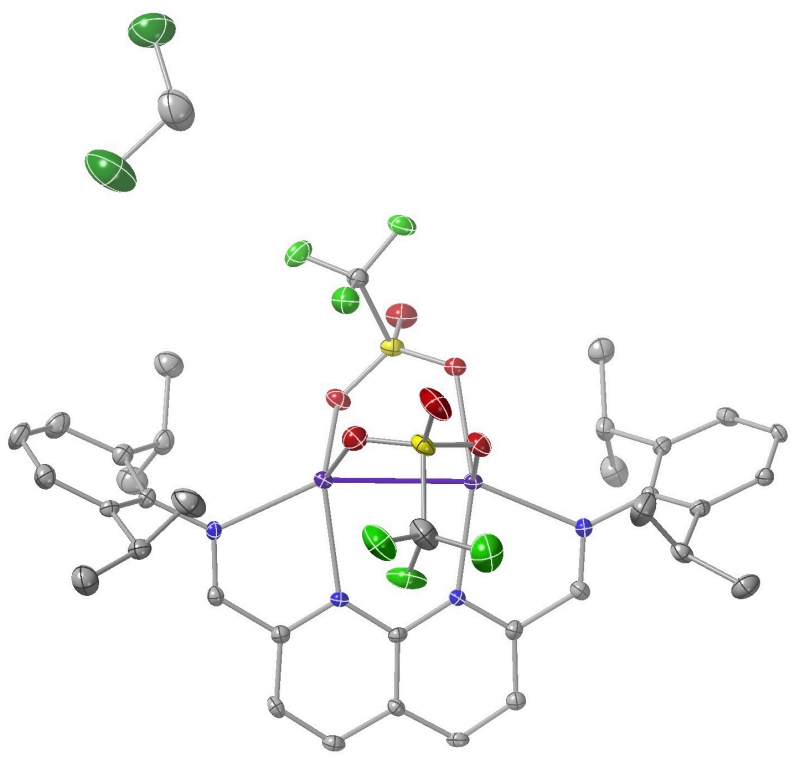

Figure S1. X-ray structure of $\mathbf{2}$, plotted with $50 \%$ probability ellipsoids ( $\mathrm{H}$ atoms omitted for clarity).

Table S1. Details for SCXRD Analysis of 2

\begin{tabular}{|l|l|}
\hline \multicolumn{2}{|l|}{ Crystal data } \\
\hline Chemical formula & $\mathrm{C}_{36} \mathrm{H}_{40} \mathrm{Ag}_{2} \mathrm{~F}_{6} \mathrm{~N}_{4} \mathrm{O}_{6} \mathrm{~S}_{2} \cdot \mathrm{CH}_{2} \mathrm{Cl}_{2}$ \\
\hline$M r$ & 1103.50 \\
\hline
\end{tabular}




\begin{tabular}{|c|c|}
\hline Crystal system, space group & Monoclinic, $P 2_{1} / n$ \\
\hline Temperature (K) & 100 \\
\hline$a, b, c(\AA)$ & 18.9640 (6), 9.7223 (3), 23.4963 (7) \\
\hline$\beta\left(^{\circ}\right)$ & $97.636(3)$ \\
\hline$V\left(\AA^{3}\right)$ & $4293.7(2)$ \\
\hline Z & 4 \\
\hline Radiation type & Mo $K \alpha$ \\
\hline$\mu\left(\mathrm{mm}^{-1}\right)$ & 1.21 \\
\hline \multicolumn{2}{|l|}{ Data collection } \\
\hline Diffractometer & SuperNova, Dual, Cu at zero, EosS2 \\
\hline Absorption correction & $\begin{array}{l}\text { Multi-scan CrysAlis PRO 1.171.38.46 (Rigaku } \\
\text { Oxford Diffraction, 2015) Empirical absorption } \\
\text { correction using spherical harmonics, implemented } \\
\text { in SCALE3 ABSPACK scaling algorithm. }\end{array}$ \\
\hline$T_{\min }, T_{\max }$ & $0.813,1.000$ \\
\hline $\begin{array}{l}\text { No. of measured, } \\
\text { independent and observed } \\
{[I>2 \sigma(I)] \text { reflections }}\end{array}$ & $42330,10613,8720$ \\
\hline$R_{\text {int }}$ & 0.060 \\
\hline$(\sin \theta / \lambda)_{\max }\left(\AA^{-1}\right)$ & 0.697 \\
\hline \multicolumn{2}{|l|}{ Refinement } \\
\hline$R\left[F^{2}>2 \sigma\left(F^{2}\right)\right], w R\left(F^{2}\right), S$ & $0.054,0.117,1.08$ \\
\hline No. of reflections & 10613 \\
\hline No. of parameters & 540 \\
\hline $\mathrm{H}$-atom treatment & $\mathrm{H}$-atom parameters constrained \\
\hline$\Delta \rho_{\max }, \Delta \rho_{\min }\left(\mathrm{e} \AA^{-3}\right)$ & $1.70,-1.56$ \\
\hline
\end{tabular}

Computer programs: CrysAlis PRO 1.171.38.46 (Rigaku OD, 2015), SHELXT (Sheldrick, 2015), SHELXL (Sheldrick, 2015), Olex2 (Dolomanov et al., 2009). 
[('PrNDI) $\left.)_{2} \mathrm{Ag}\right][\mathrm{OTf}](3)(\mathrm{CCDC}$ 1997403)

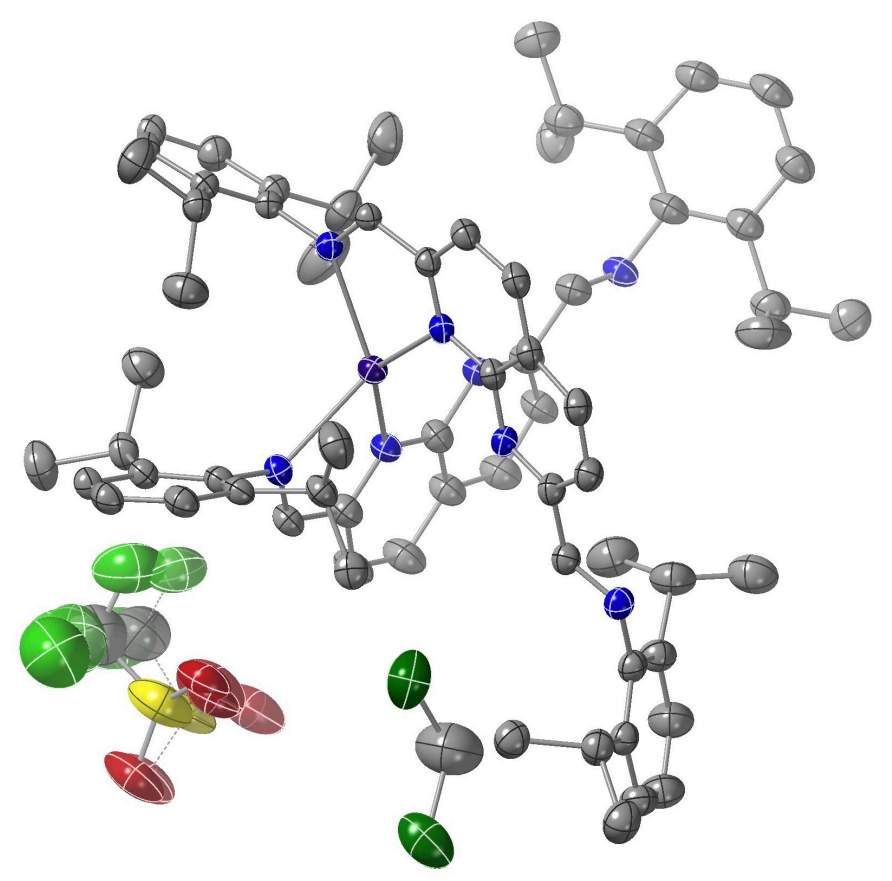

Figure S2. X-ray structure of 3 , plotted with $50 \%$ probability ellipsoids ( $\mathrm{H}$ atoms omitted for clarity). The disorder model is shown using transparent ellipsoids and dashed bonds.

Table S2. Details for SCXRD Analysis of 3

\begin{tabular}{|c|c|}
\hline \multicolumn{2}{|l|}{ Crystal data } \\
\hline Chemical formula & $\mathrm{C}_{68} \mathrm{H}_{80} \mathrm{AgN}_{8} \cdot \mathrm{CH}_{2} \mathrm{Cl}_{2} \cdot \mathrm{CF}_{3} \mathrm{SO}_{3}$ \\
\hline Mr & 1351.26 \\
\hline Crystal system, space group & Orthorhombic, Pbca \\
\hline Temperature $(\mathrm{K})$ & 100 \\
\hline$a, b, c(\AA)$ & 27.7976 (4), $17.5888(2), 31.1117(4)$ \\
\hline$V\left(\AA^{3}\right)$ & $15211.3(3)$ \\
\hline $\begin{array}{l}Z \\
\end{array}$ & 8 \\
\hline Radiation type & $\mathrm{Cu} K \alpha$ \\
\hline$\mu\left(\mathrm{mm}^{-1}\right)$ & 3.46 \\
\hline \multicolumn{2}{|l|}{ Data collection } \\
\hline Diffractometer & SuperNova, Dual, Cu at zero, EosS2 \\
\hline Absorption correction & $\begin{array}{l}\text { Multi-scan } \\
\text { CrysAlis PRO 1.171.38.46 (Rigaku Oxford Diffraction, 2015) } \\
\text { Empirical absorption correction using spherical harmonics, }\end{array}$ \\
\hline
\end{tabular}




\begin{tabular}{|l|l|}
\hline & implemented in SCALE3 ABSPACK scaling algorithm. \\
\hline$T_{\min }, T_{\max }$ & $0.694,1.000$ \\
\hline $\begin{array}{l}\text { No. of measured, } \\
\text { independent and } \\
\text { observed }[I>2 \sigma(I)] \\
\text { reflections }\end{array}$ & $215036,15195,12532$ \\
\hline$R_{\text {int }}$ & 0.088 \\
\hline$(\sin \theta / \lambda)_{\max }\left(\AA^{-1}\right)$ & 0.625 \\
\hline Refinement & $0.058,0.172,1.09$ \\
\hline$R\left[F^{2}>2 \sigma\left(F^{2}\right)\right], w R\left(F^{2}\right), S$ & 15195 \\
\hline No. of reflections & 883 \\
\hline No. of parameters & 173 \\
\hline No. of restraints & H-atom parameters constrained \\
\hline$H$-atom treatment & $1.13,-0.91$ \\
\hline$\Delta \rho_{\max }, \Delta \rho_{\min }\left(\mathrm{e} \AA^{-3}\right)$ &
\end{tabular}

Computer programs: CrysAlis PRO 1.171.38.46 (Rigaku OD, 2015), SHELXL 2018/3 (Sheldrick, 2015), Olex2 1.3 (Dolomanov et al., 2009). 


\section{$\left[\left({ }^{\prime} \mathrm{PrNDI}\right)_{2} \mathrm{Ag}_{2}\right][\mathrm{OTf}]_{2}(4 \cdot \mathrm{OTf})(\mathrm{CCDC} 1997404)$}

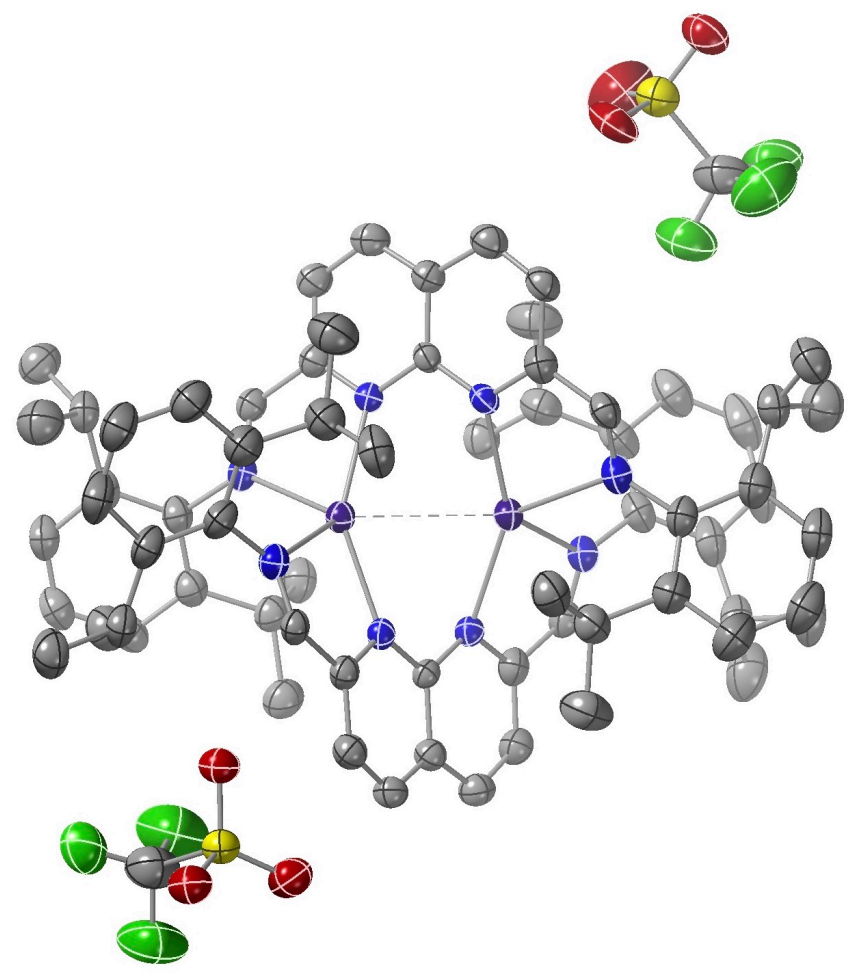

Figure S3. X-ray structure of $4 \cdot 0$ Tf, plotted with $50 \%$ probability ellipsoids $(\mathrm{H}$ atoms omitted for clarity).

Table S3. Details for SCXRD Analysis of 4•OTf

\begin{tabular}{|c|c|}
\hline \multicolumn{2}{|l|}{ Crystal data } \\
\hline Chemical formula & $\mathrm{C}_{68} \mathrm{H}_{80} \mathrm{Ag}_{2} \mathrm{~N}_{8} \cdot 2\left(\mathrm{CF}_{3} \mathrm{O}_{3} \mathrm{~S}\right)$ \\
\hline$M r$ & 1523.28 \\
\hline Crystal system, space group & Monoclinic, C2/c \\
\hline \begin{tabular}{|l|l} 
Temperature (K) \\
\end{tabular} & 100 \\
\hline$a, b, c(\AA)$ & $56.595(2), 16.9517(4), 19.7131(8)$ \\
\hline$\beta\left(^{\circ}\right)$ & $121.094(6)$ \\
\hline$V\left(\AA^{3}\right)$ & $16195.1(13)$ \\
\hline$Z$ & 8 \\
\hline Radiation type & $\mathrm{Cu} K \alpha$ \\
\hline$\mu\left(\mathrm{mm}^{-1}\right)$ & 4.89 \\
\hline \multicolumn{2}{|l|}{ Data collection } \\
\hline Diffractometer & SuperNova, Dual, Cu at zero, EosS2 \\
\hline
\end{tabular}




\begin{tabular}{|c|c|}
\hline Absorption correction & $\begin{array}{l}\text { Gaussian } \\
\text { CrysAlis PRO 1.171.38.46 (Rigaku Oxford Diffraction, 2015) } \\
\text { Numerical absorption correction based on gaussian } \\
\text { integration over a multifaceted crystal model } \\
\text { Empirical absorption correction using spherical harmonics, } \\
\text { implemented in SCALE3 ABSPACK scaling algorithm. }\end{array}$ \\
\hline$T_{\min }, T_{\max }$ & $0.764,0.876$ \\
\hline $\begin{array}{l}\text { No. of measured, } \\
\text { independent and } \\
\text { observed }[I>2 \sigma(I)] \\
\text { reflections }\end{array}$ & $96819,16079,11446$ \\
\hline$R_{\text {int }}$ & 0.090 \\
\hline$(\sin \theta / \lambda)_{\max }\left(\AA^{-1}\right)$ & 0.620 \\
\hline \multicolumn{2}{|l|}{ Refinement } \\
\hline$R\left[F^{2}>2 \sigma\left(F^{2}\right)\right], w R\left(F^{2}\right), S$ & $0.072,0.212,1.07$ \\
\hline No. of reflections & 16079 \\
\hline No. of parameters & 863 \\
\hline $\mathrm{H}$-atom treatment & $\mathrm{H}$-atom parameters constrained \\
\hline$\Delta \rho_{\max }, \Delta \rho_{\min }\left(\mathrm{e} \AA^{-3}\right)$ & $2.17,-1.00$ \\
\hline
\end{tabular}

Computer programs: CrysAlis PRO 1.171.38.46 (Rigaku OD, 2015), SHELXT 2014/5 (Sheldrick, 2014), SHELXL 2018/3 (Sheldrick, 2015), Olex2 1.3 (Dolomanov et al., 2009). 
$\left[\left({ }^{i} \mathrm{PrNDI}\right)_{2} \mathrm{Ag}_{2}\right]\left[\mathrm{PF}_{6}\right]_{2}\left(4 \cdot \mathrm{PF}_{6}\right)(\mathrm{CCDC} 2022245)$

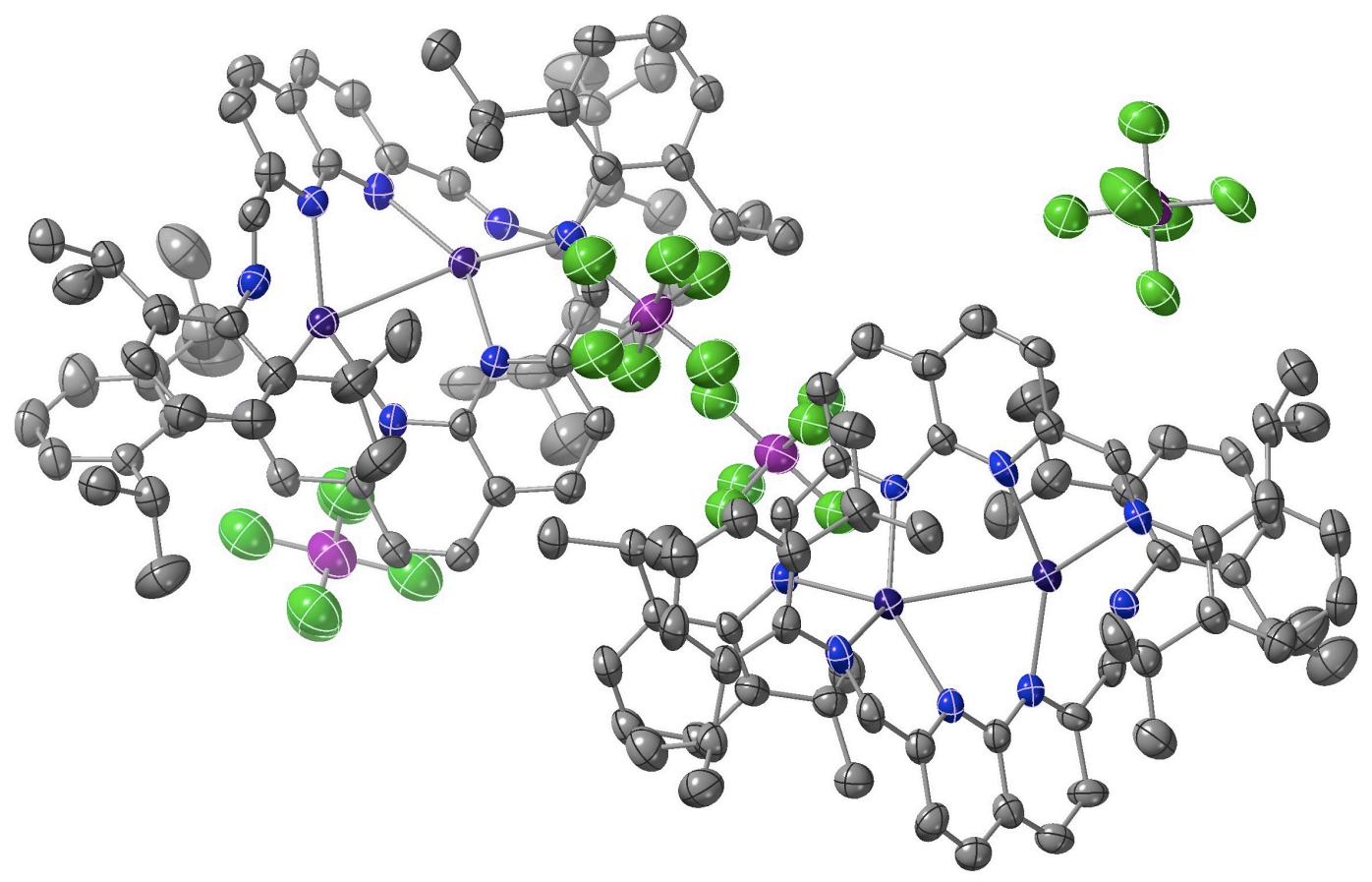

Figure S4. X-ray structure of $\mathbf{4} \cdot \mathrm{PF}_{\mathbf{6}}$, plotted with $50 \%$ probability ellipsoids $(\mathrm{H}$ atoms omitted for clarity). The asymmetric unit contains two independent molecules of $\mathbf{4} \cdot \mathbf{P F}_{\mathbf{6}}$.

Table S4. Details for SCXRD Analysis of $4 \cdot \mathbf{P F}_{6}$

\begin{tabular}{|c|c|}
\hline \multicolumn{2}{|l|}{ Crystal data } \\
\hline Chemical formula & $\mathrm{C}_{68} \mathrm{H}_{80} \mathrm{Ag}_{2} \mathrm{~N}_{8} \cdot 2\left(\mathrm{~F}_{6} \mathrm{P}\right)$ \\
\hline$M_{\mathrm{r}}$ & 1515.08 \\
\hline Crystal system, space group & Triclinic, $P^{-} 1$ \\
\hline Temperature (K) & 100 \\
\hline$a, b, c(\AA)$ & $16.8734(4), 20.5537(6), 23.1457(7)$ \\
\hline$\alpha, \beta, \gamma\left({ }^{\circ}\right)$ & $65.559(3), 83.043(2), 88.895(2)$ \\
\hline$V\left(\AA^{3}\right)$ & $7249.8(4)$ \\
\hline$Z$ & 4 \\
\hline Radiation type & $\mathrm{Cu} K \alpha$ \\
\hline$\mu\left(\mathrm{mm}^{-1}\right)$ & 5.39 \\
\hline \multicolumn{2}{|l|}{ Data collection } \\
\hline Diffractometer & SuperNova, Dual, Cu at zero, EosS2 \\
\hline Absorption correction & Multi-scan \\
\hline
\end{tabular}




\begin{tabular}{|c|c|}
\hline & $\begin{array}{l}\text { CrysAlis PRO 1.171.38.46 (Rigaku Oxford } \\
\text { Diffraction, 2015) Empirical absorption correction } \\
\text { using spherical harmonics, implemented in } \\
\text { SCALE3 ABSPACK scaling algorithm. }\end{array}$ \\
\hline$T_{\min }, T_{\max }$ & $0.873,1.000$ \\
\hline $\begin{array}{l}\text { No. of measured, } \\
\text { independent and } \\
\text { observed }[I>2 \sigma(I)] \\
\text { reflections }\end{array}$ & $57158,28419,18563$ \\
\hline$R_{\text {int }}$ & 0.063 \\
\hline$(\sin \theta / \lambda)_{\max }\left(\AA^{-1}\right)$ & 0.620 \\
\hline \multicolumn{2}{|l|}{ Refinement } \\
\hline$R\left[F^{2}>2 \sigma\left(F^{2}\right)\right], w R\left(F^{2}\right), S$ & $0.075,0.227,1.03$ \\
\hline No. of reflections & 28419 \\
\hline No. of parameters & 1587 \\
\hline $\mathrm{H}$-atom treatment & $\mathrm{H}$-atom parameters constrained \\
\hline$\Delta \rho_{\max }, \Delta \rho_{\min }\left(\mathrm{e} \AA^{-3}\right)$ & $2.05,-1.33$ \\
\hline
\end{tabular}

Computer programs: CrysAlis PRO 1.171.38.46 (Rigaku OD, 2015), SHELXT 2014/5 (Sheldrick, 2014), SHELXL (Sheldrick, 2015), Olex2 (Dolomanov et al., 2009).

\section{UV-vis Data}

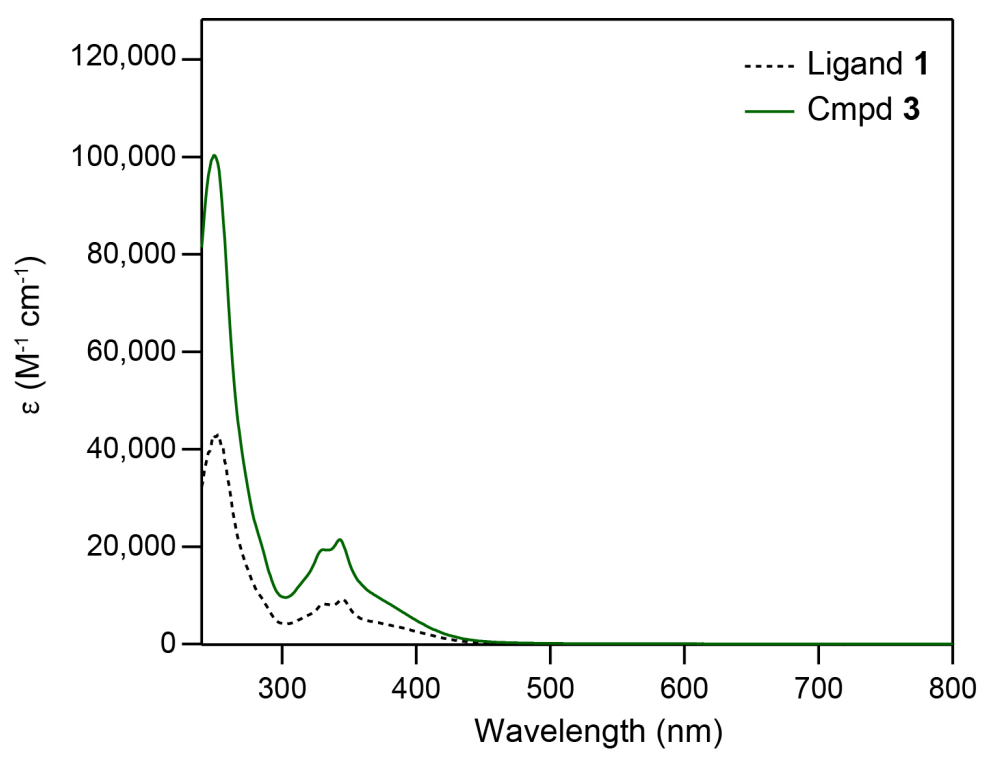

Figure S5. UV-vis spectrum of 3, overlaid with the spectrum of ligand 1. 


\section{LFP Data}

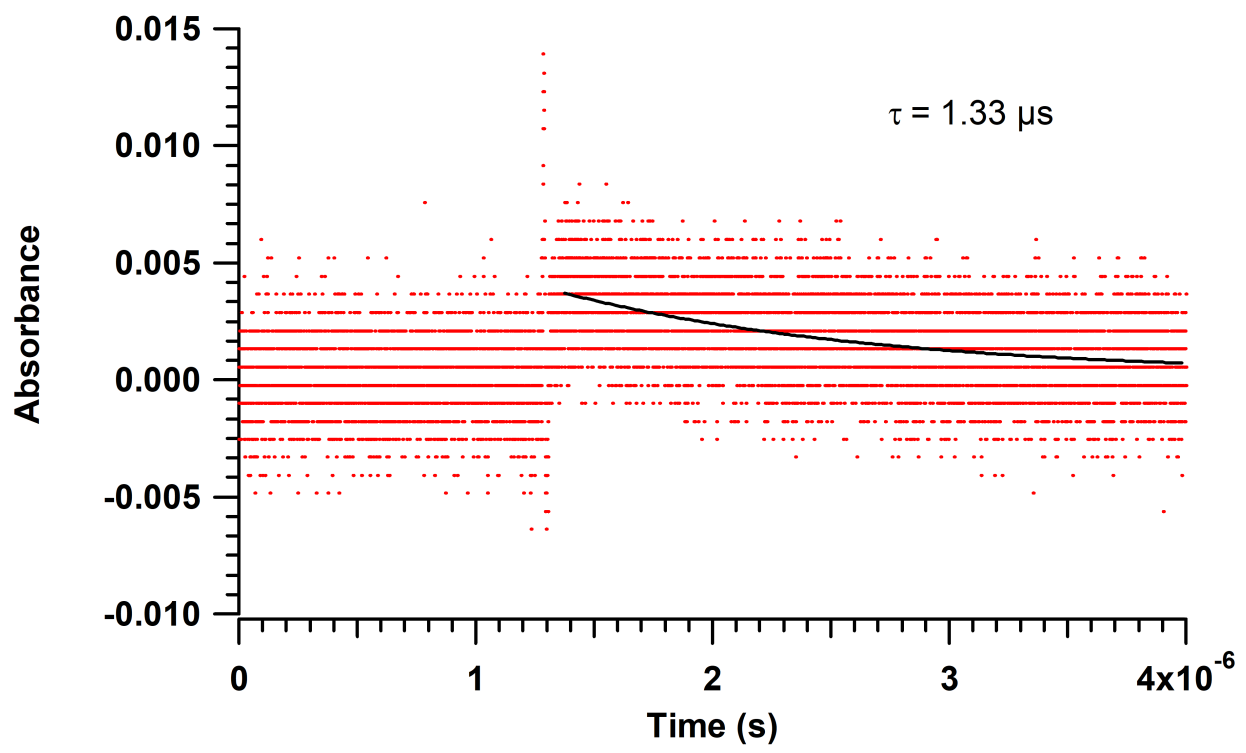

Figure S6. Representative kinetic trace at $500 \mathrm{~nm}$ for LFP of 2 in $\mathrm{CH}_{2} \mathrm{Cl}_{2}$; the solid black line corresponds to the fit of the experimental data $\left(k=7.5 \times 10^{5} \pm 0.8 \times 10^{5}, \tau=1.33 \mu \mathrm{s}\right)$.

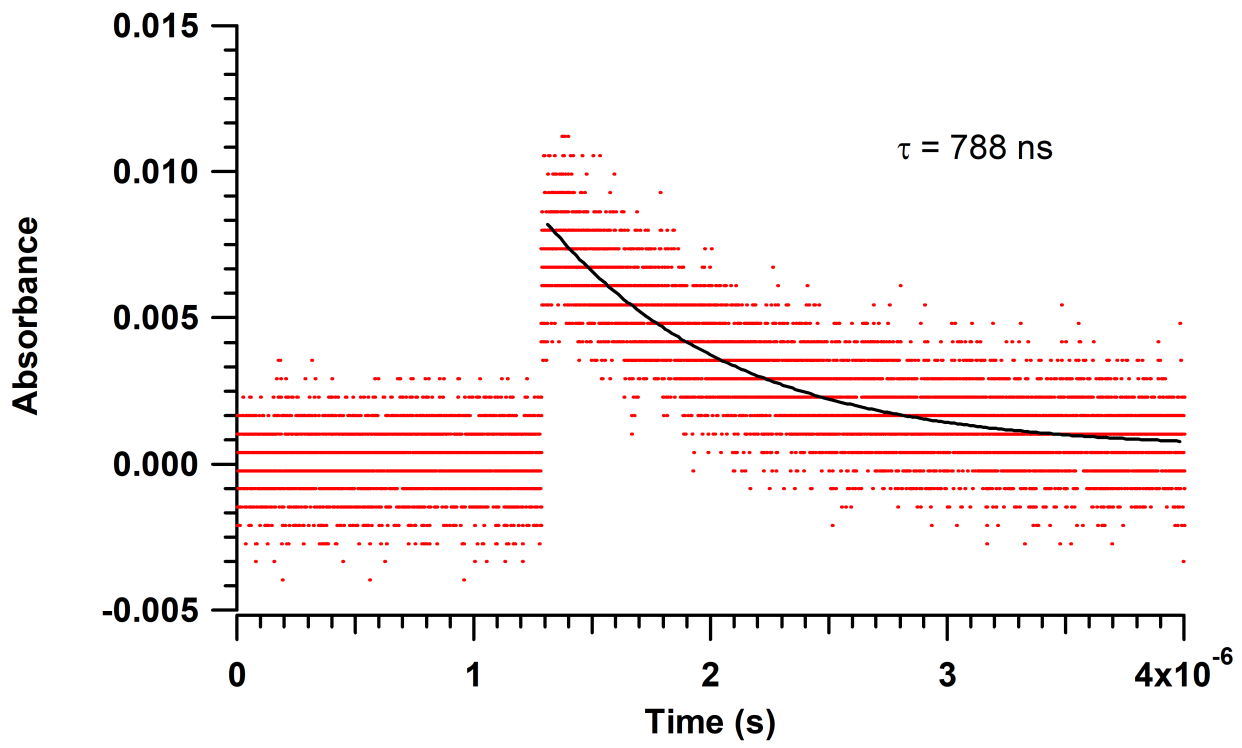

Figure S7. Representative kinetic trace at $500 \mathrm{~nm}$ for LFP of $\mathbf{4} \cdot \mathrm{PF}_{6}$ in $\mathrm{CH}_{2} \mathrm{Cl}_{2}$; the solid black line corresponds to the fit of the experimental data $\left(\mathrm{k}=1.27 \times 10^{6} \pm 0.03 \times 10^{6}, \tau=\right.$ $788 \mathrm{~ns})$. 


\section{Mass Spectrometry Data}
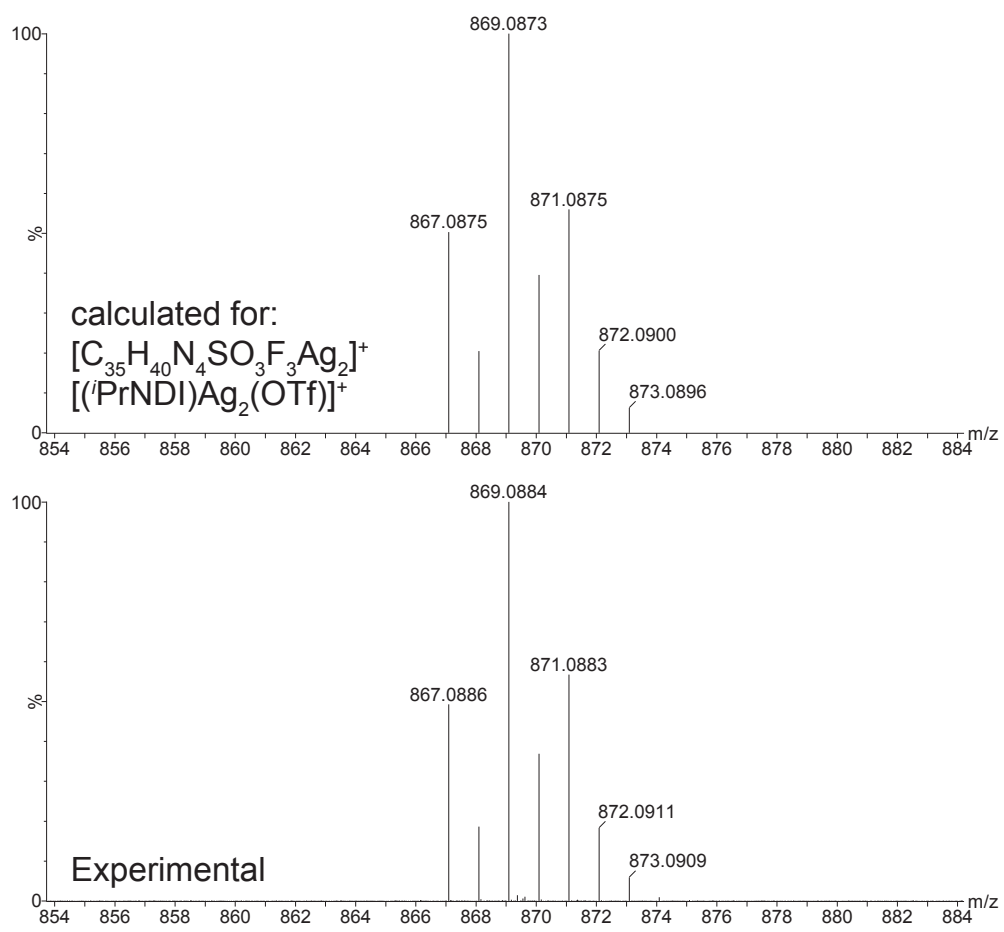

Figure S8. Predicted and experimental HRMS data (ESI+) for 2, which ionizes via loss of one triflate anion.
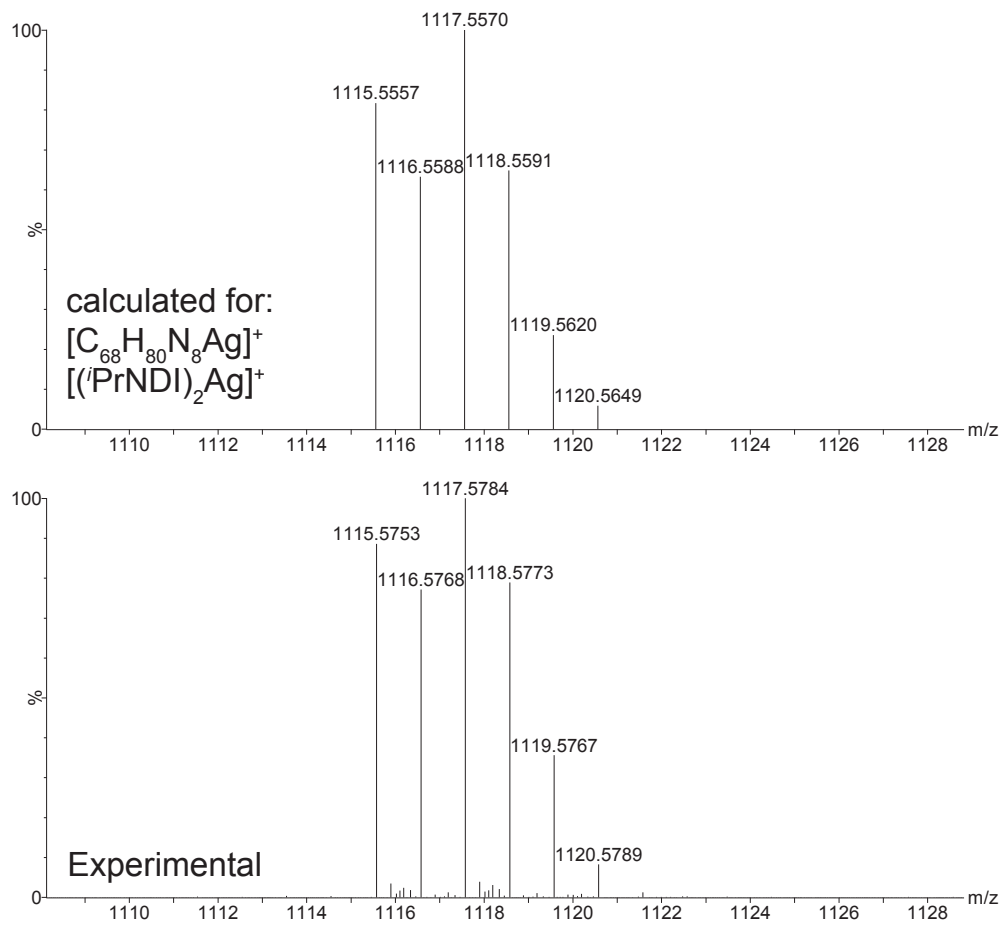

Figure S9. Predicted and experimental HRMS data (ESI+) for 3. 


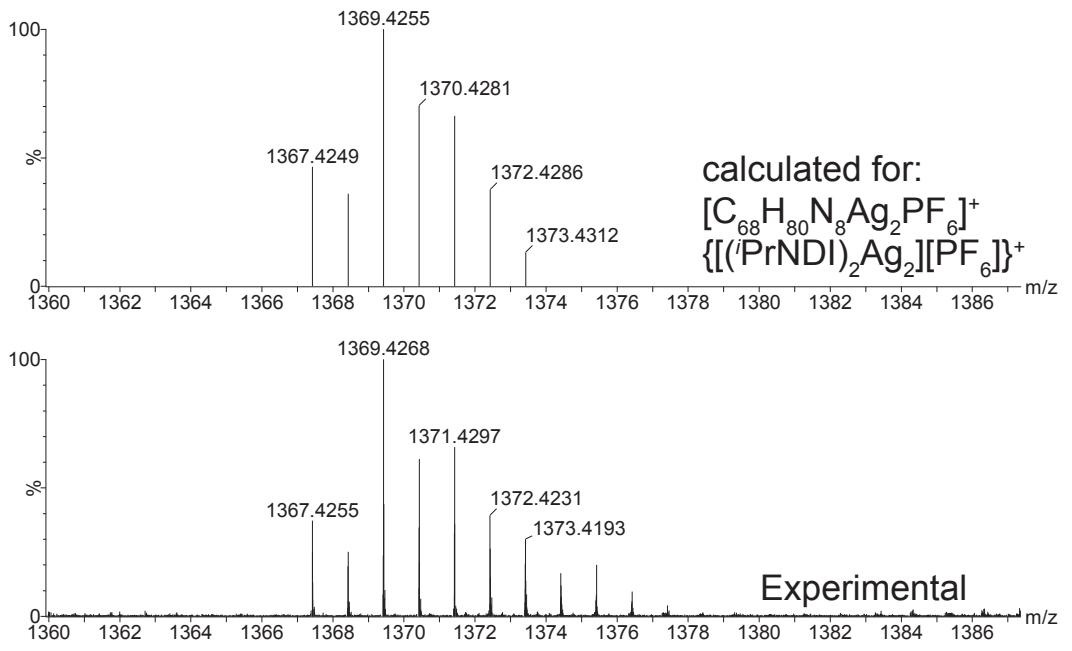

Figure S10. Predicted and experimental HRMS data $(E S I+)$ for $\mathbf{4} \cdot \mathbf{P F}_{6}$, which was observed as a monocationic ion pair with one hexafluorophosphate anion.

\section{NMR Data}

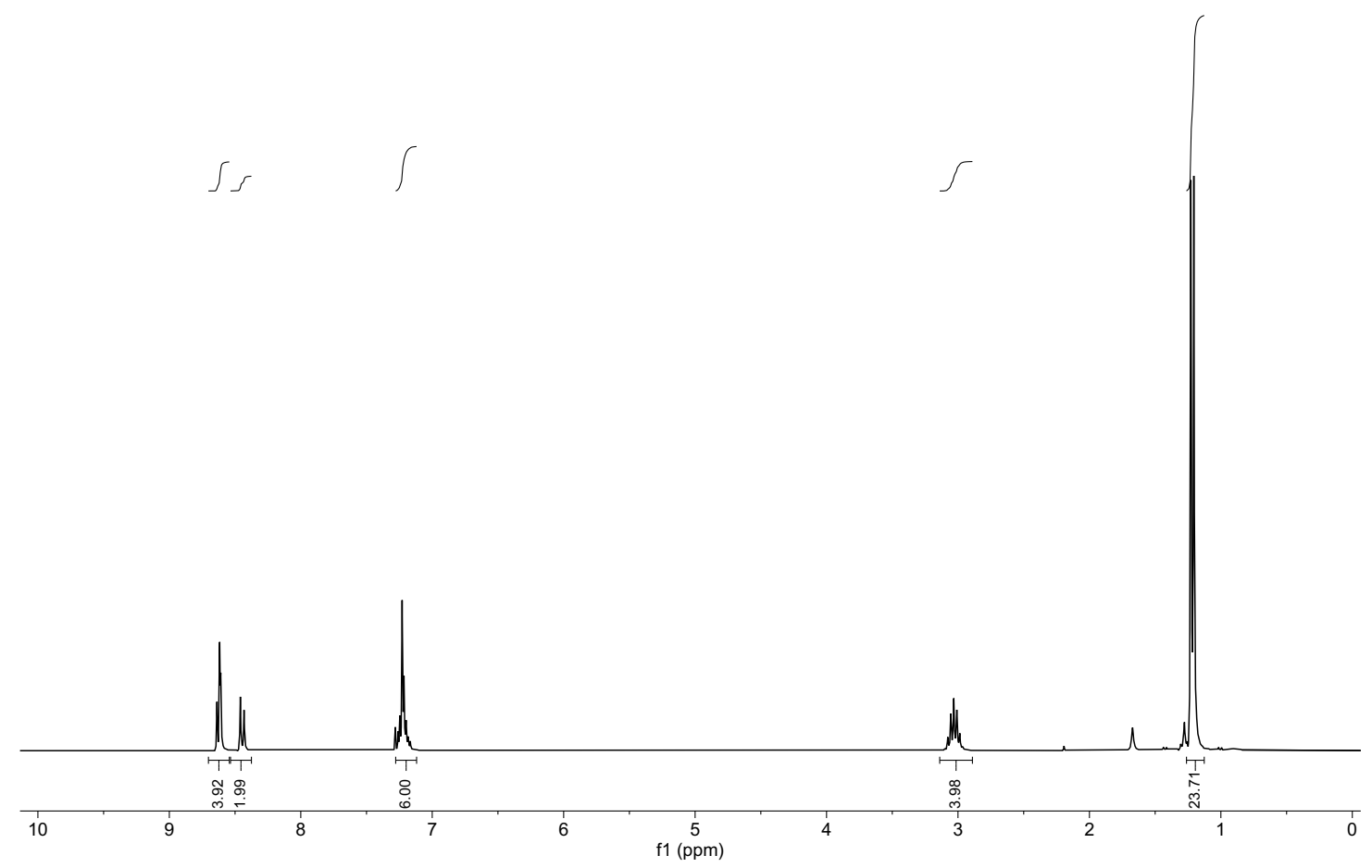

Figure S11. ${ }^{1} \mathrm{H}$ NMR spectrum for $1\left(\mathrm{CDCl}_{3}, 300 \mathrm{MHz}, 23{ }^{\circ} \mathrm{C}\right)$ 


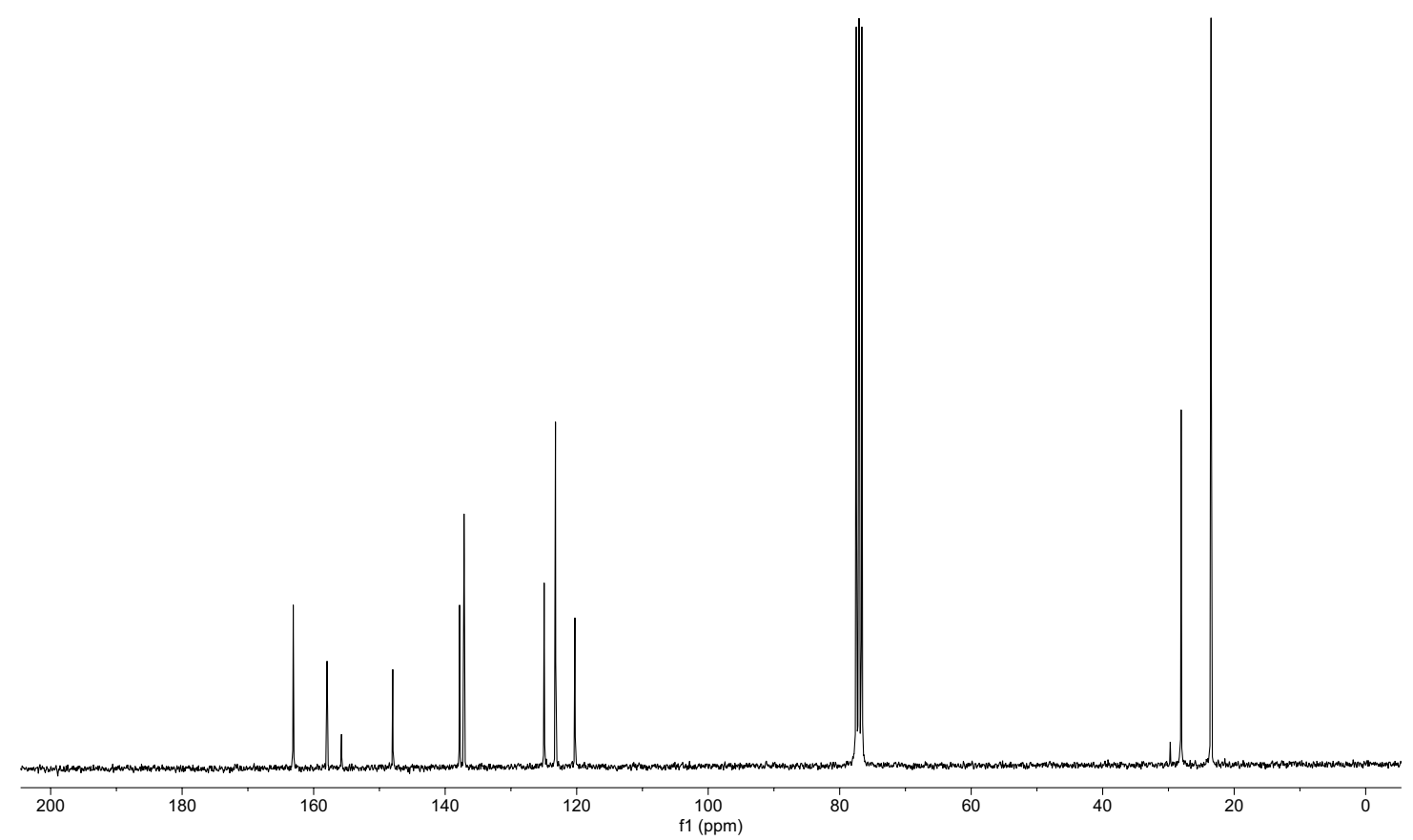

Figure S12. ${ }^{13} \mathrm{C}$ NMR spectrum for $1\left(\mathrm{CDCl}_{3}, 75 \mathrm{MHz}, 23{ }^{\circ} \mathrm{C}\right)$

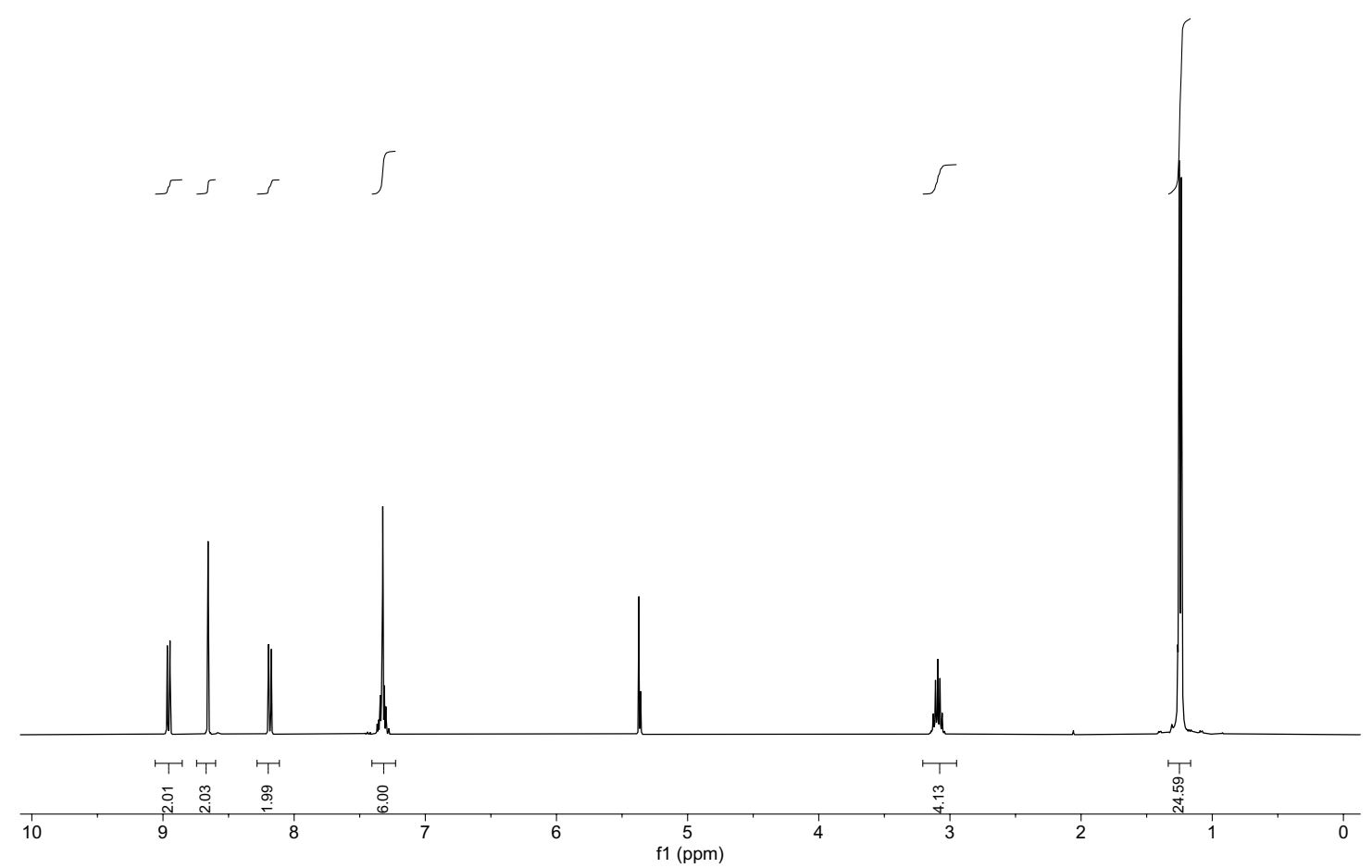

Figure S13. ${ }^{1} \mathrm{H}$ NMR spectrum for $2\left(\mathrm{CD}_{2} \mathrm{Cl}_{2}, 400 \mathrm{MHz}, 25^{\circ} \mathrm{C}\right)$ 


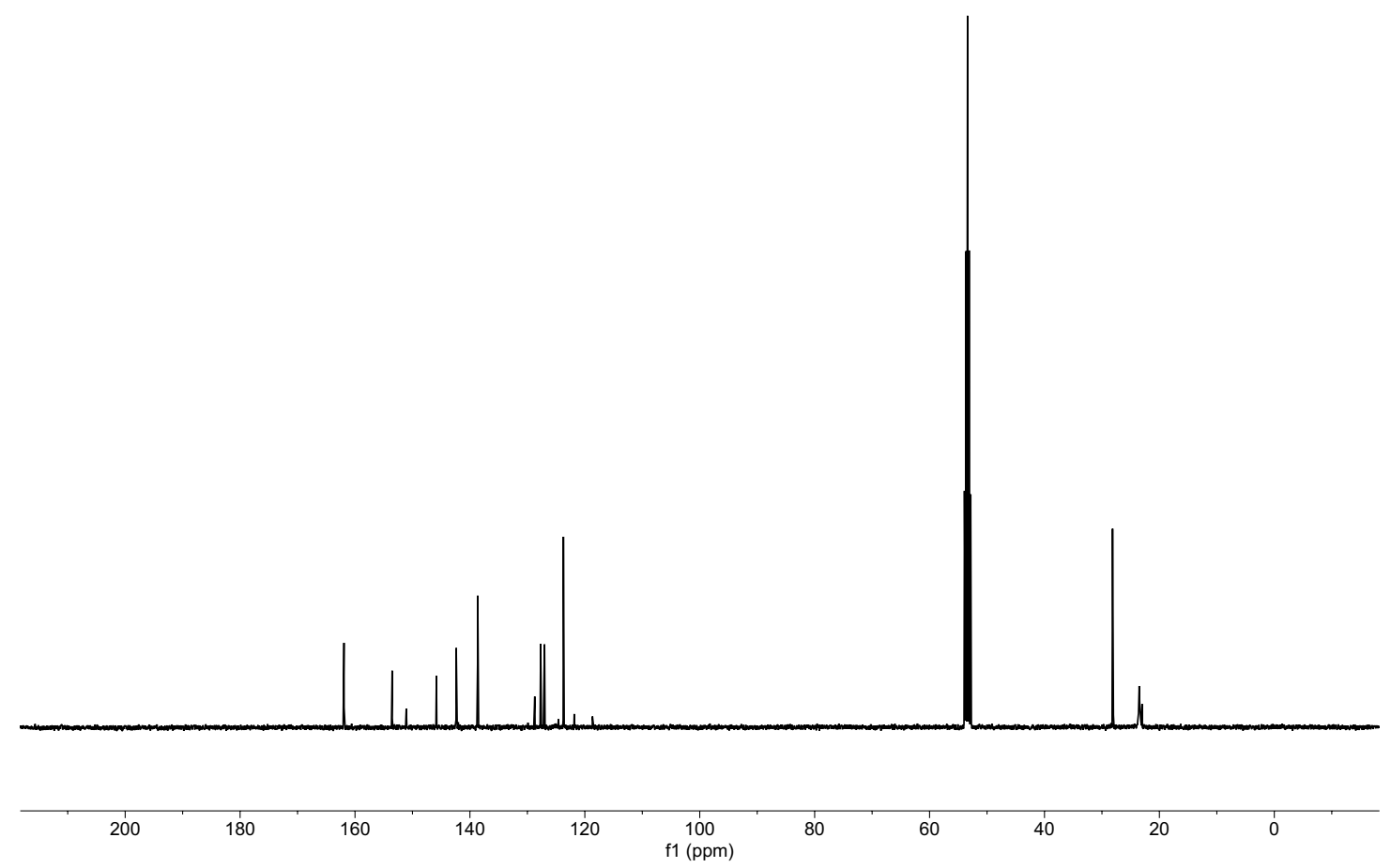

Figure S14. ${ }^{13} \mathrm{C}$ NMR spectrum for $2\left(\mathrm{CD}_{2} \mathrm{Cl}_{2}, 100 \mathrm{MHz}, 25{ }^{\circ} \mathrm{C}\right)$

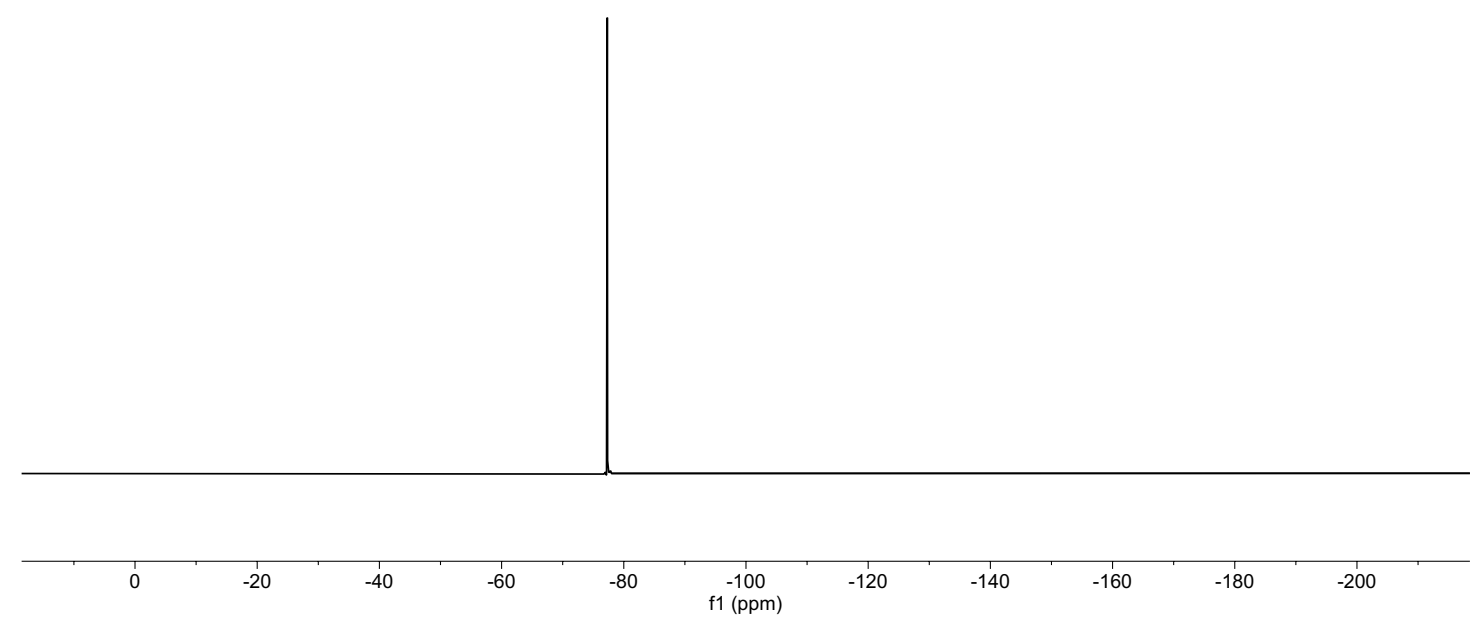

Figure S15. ${ }^{19} \mathrm{~F}$ NMR spectrum for $2\left(\mathrm{CD}_{2} \mathrm{Cl}_{2}, 376 \mathrm{MHz}, 25{ }^{\circ} \mathrm{C}\right)$ 


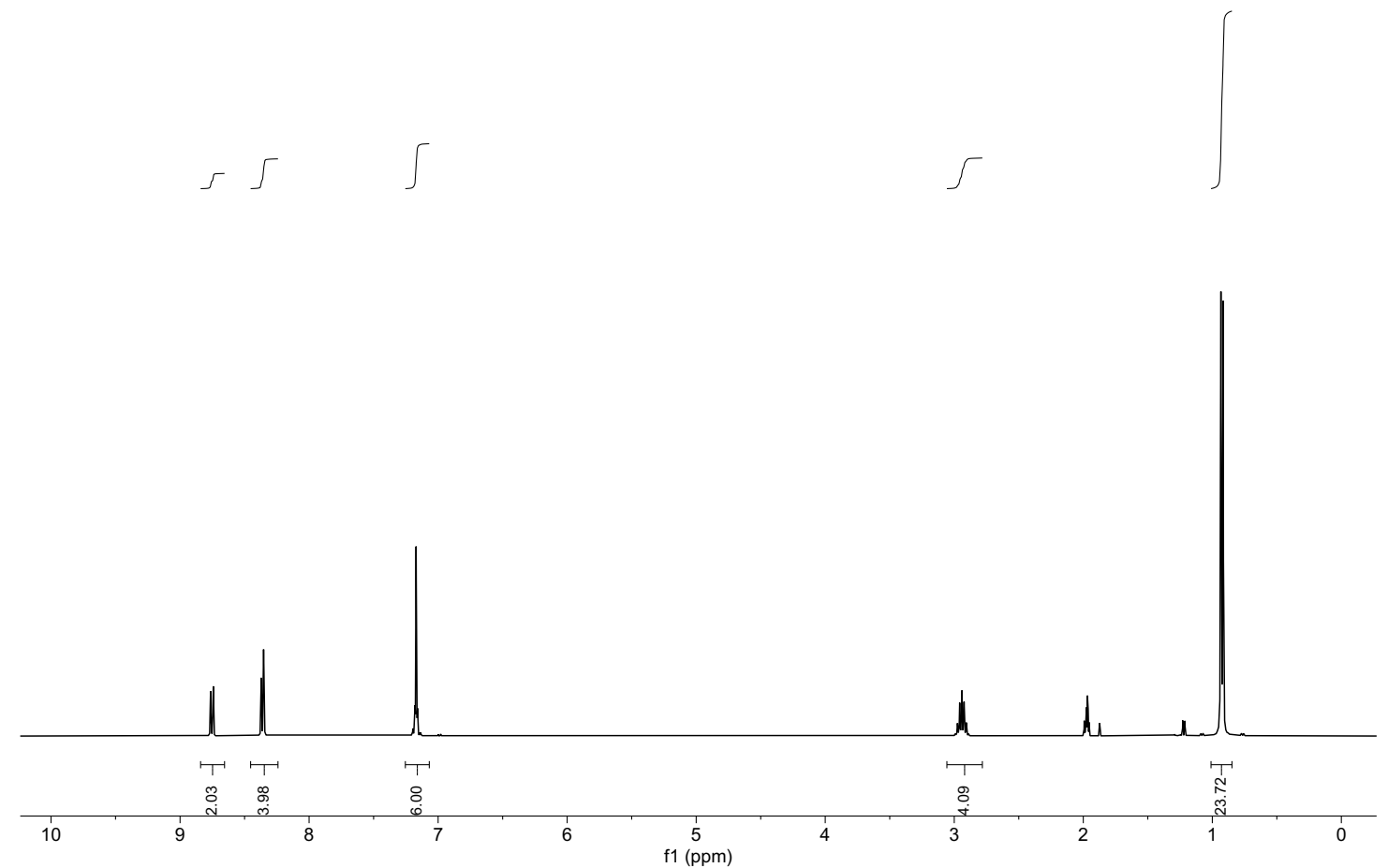

Figure S16. ${ }^{1} \mathrm{H}$ NMR spectrum for $3\left(\mathrm{CD}_{3} \mathrm{CN}, 400 \mathrm{MHz}, 25^{\circ} \mathrm{C}\right)$

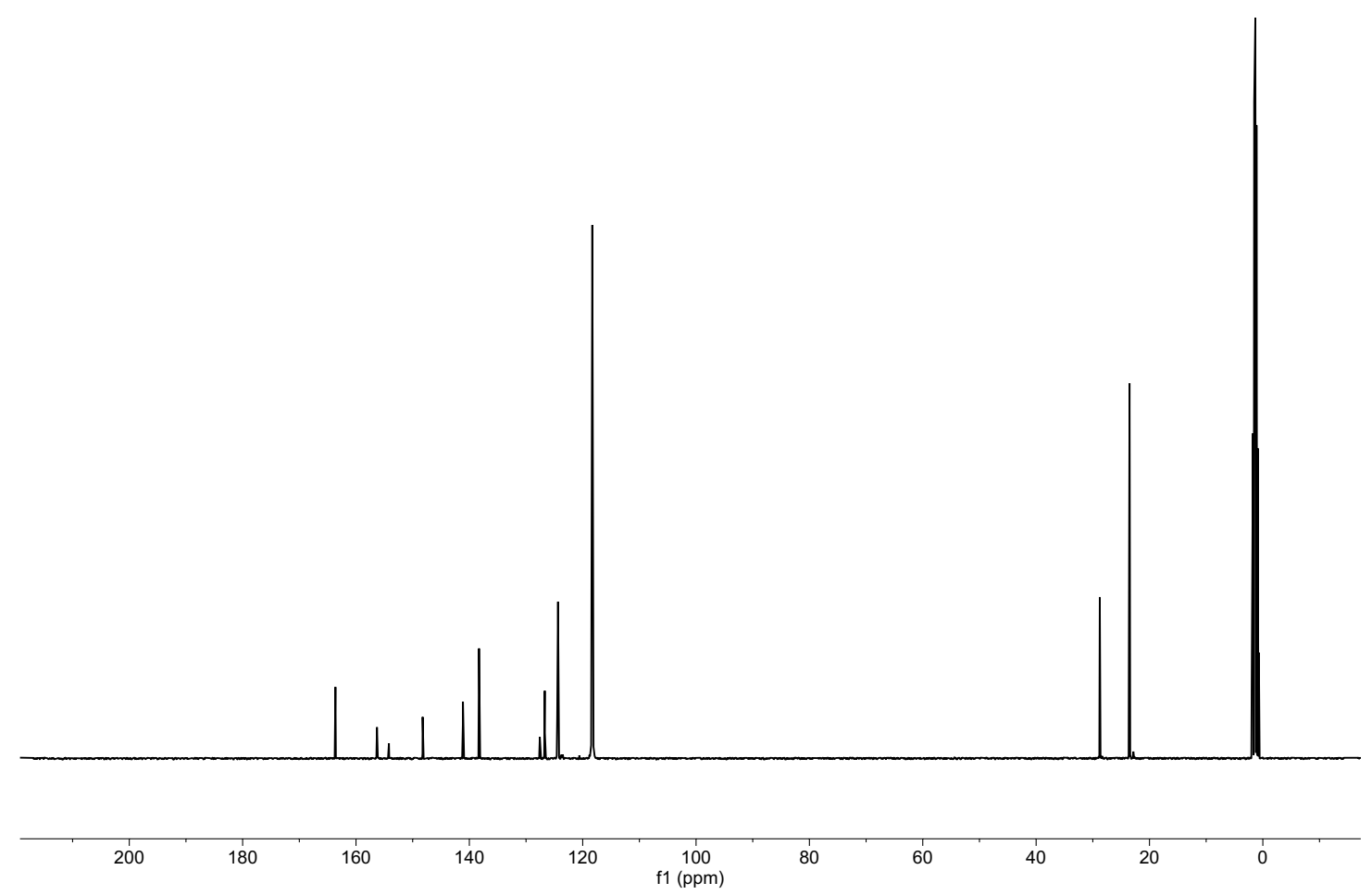

Figure S17. ${ }^{13} \mathrm{C}$ NMR spectrum for $3\left(\mathrm{CD}_{3} \mathrm{CN}, 100 \mathrm{MHz}, 25{ }^{\circ} \mathrm{C}\right)$ 


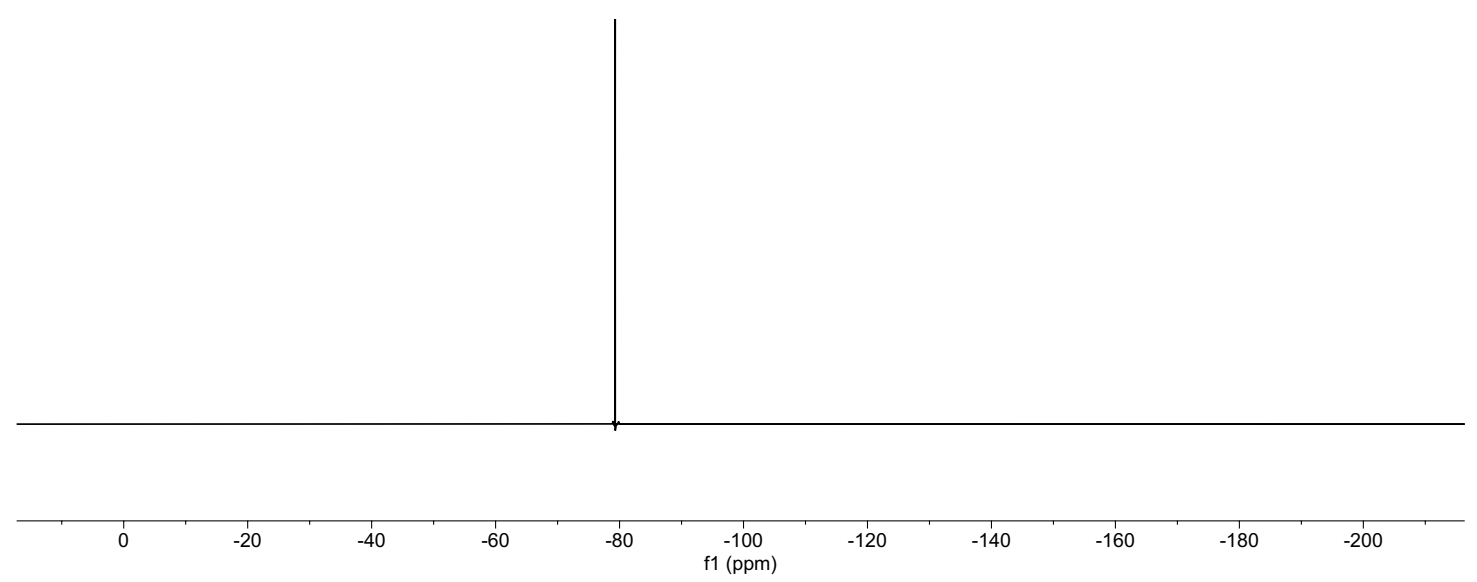

Figure S18. ${ }^{19} \mathrm{~F}$ NMR spectrum for $3\left(\mathrm{CD}_{3} \mathrm{CN}, 376 \mathrm{MHz}, 25{ }^{\circ} \mathrm{C}\right)$

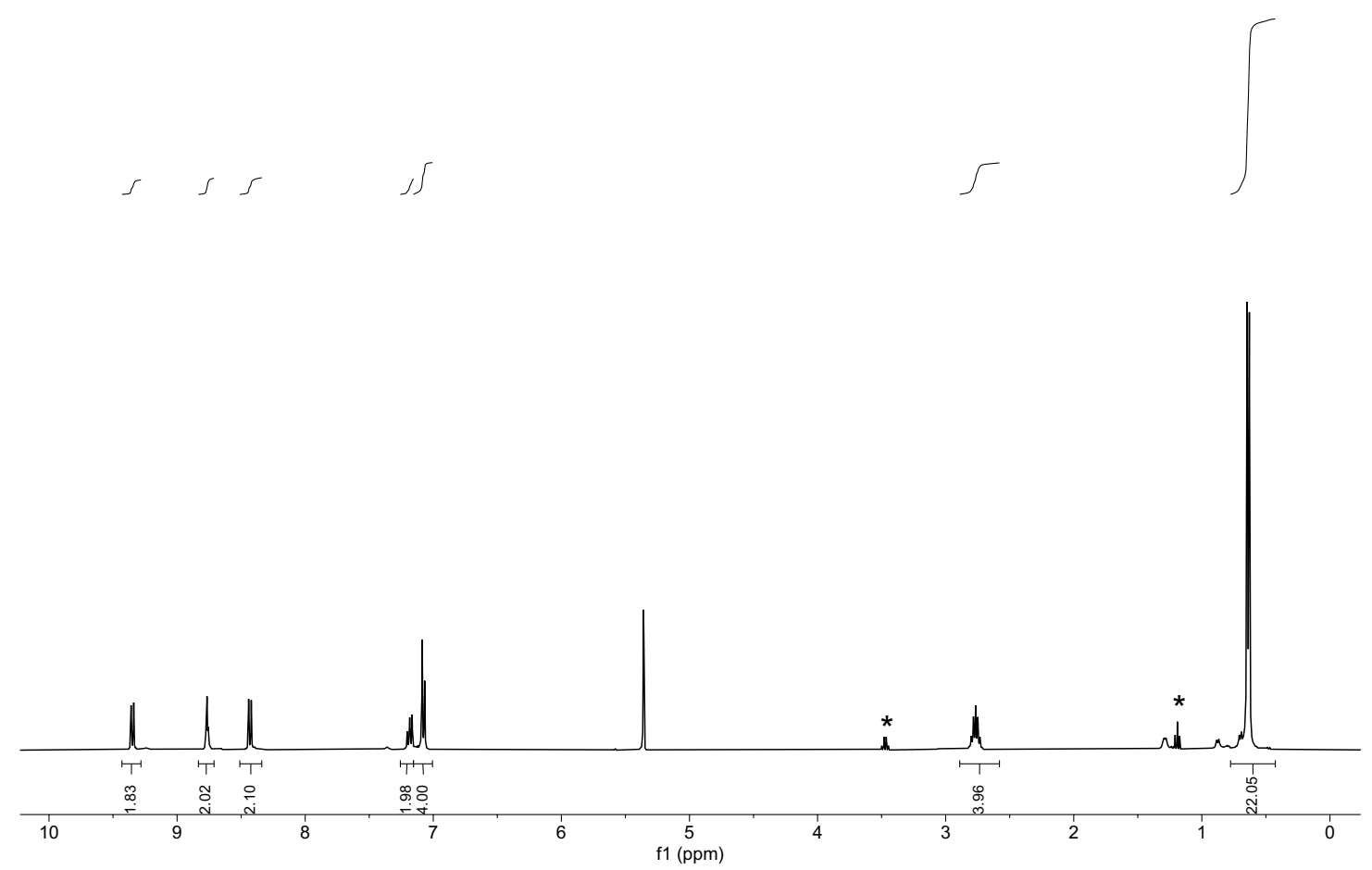

Figure S19. ${ }^{1} \mathrm{H}$ NMR spectrum for $4 \cdot \mathrm{PF}_{6}\left(\mathrm{CD}_{2} \mathrm{Cl}_{2}, 400 \mathrm{MHz}, 25^{\circ} \mathrm{C}\right)$. Asterisks ( $\left.{ }^{*}\right)$ indicate residual $\mathrm{Et}_{2} \mathrm{O}$ from recrystallization. 


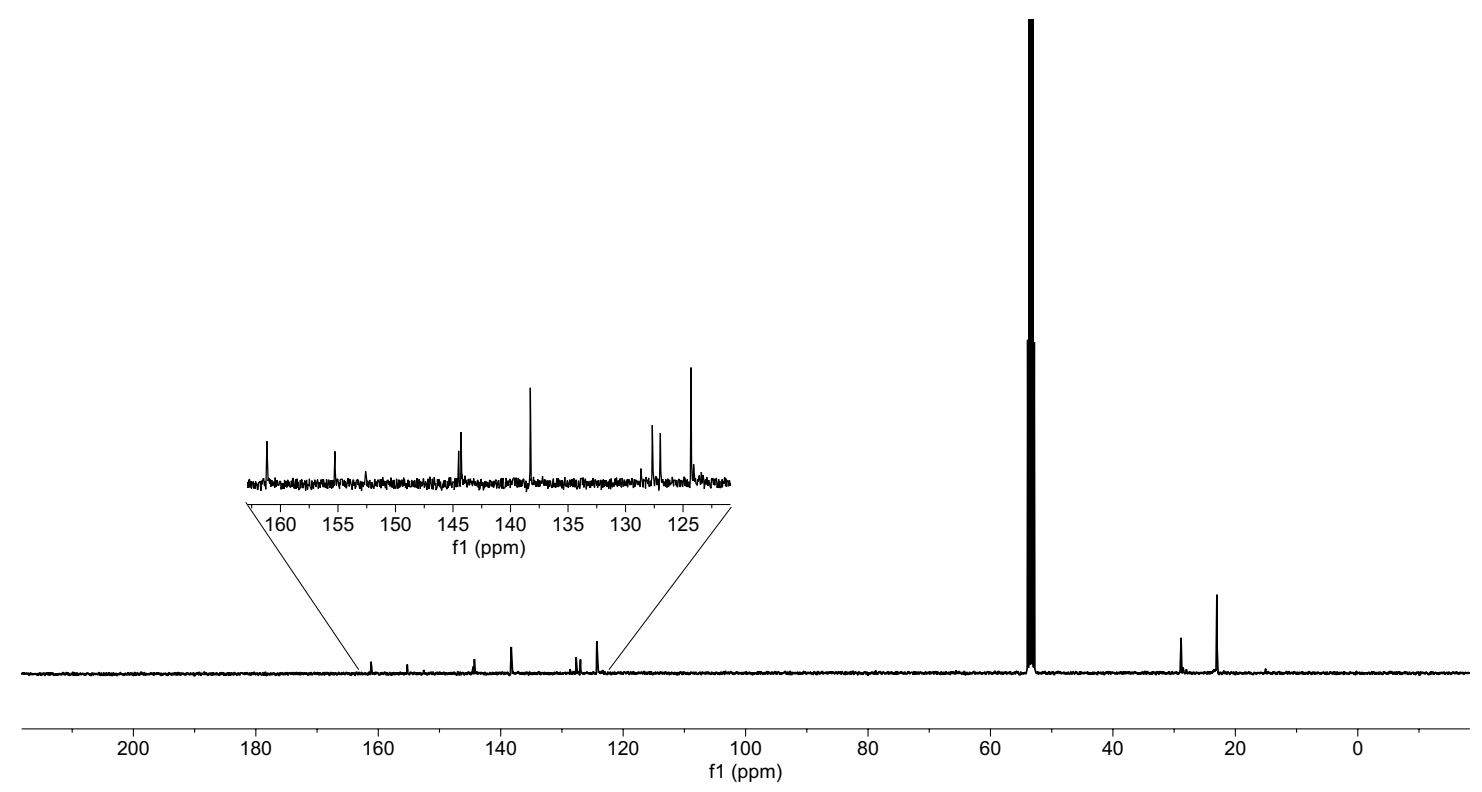

Figure S20. ${ }^{13} \mathrm{C}$ NMR spectrum for $4 \cdot \mathrm{PF}_{6}\left(\mathrm{CD}_{2} \mathrm{Cl}_{2}, 100 \mathrm{MHz}, 25^{\circ} \mathrm{C}\right)$

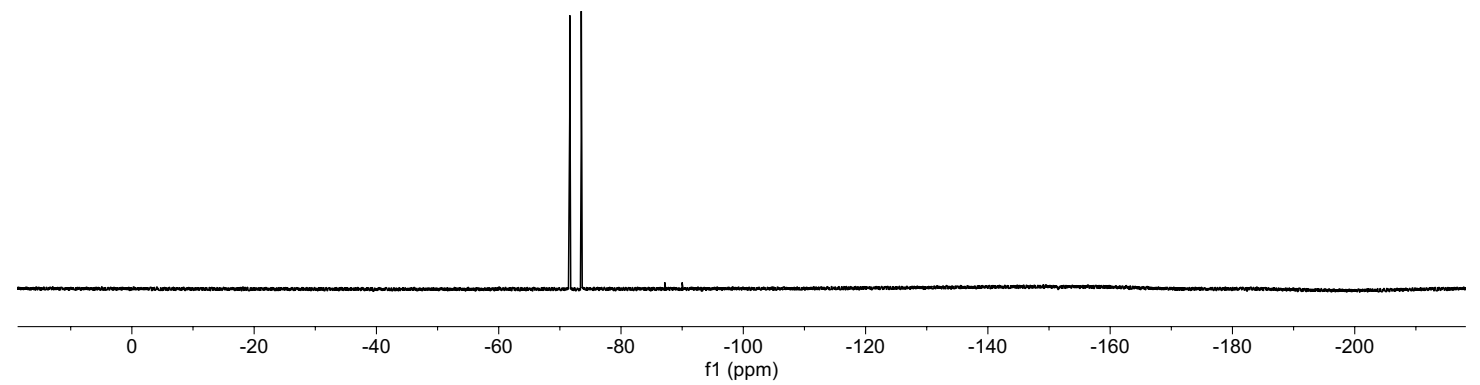

Figure S21. ${ }^{19} \mathrm{~F}$ NMR spectrum for $4 \cdot \mathrm{PF}_{6}\left(\mathrm{CD}_{2} \mathrm{Cl}_{2}, 376 \mathrm{MHz}, 25^{\circ} \mathrm{C}\right)$ 


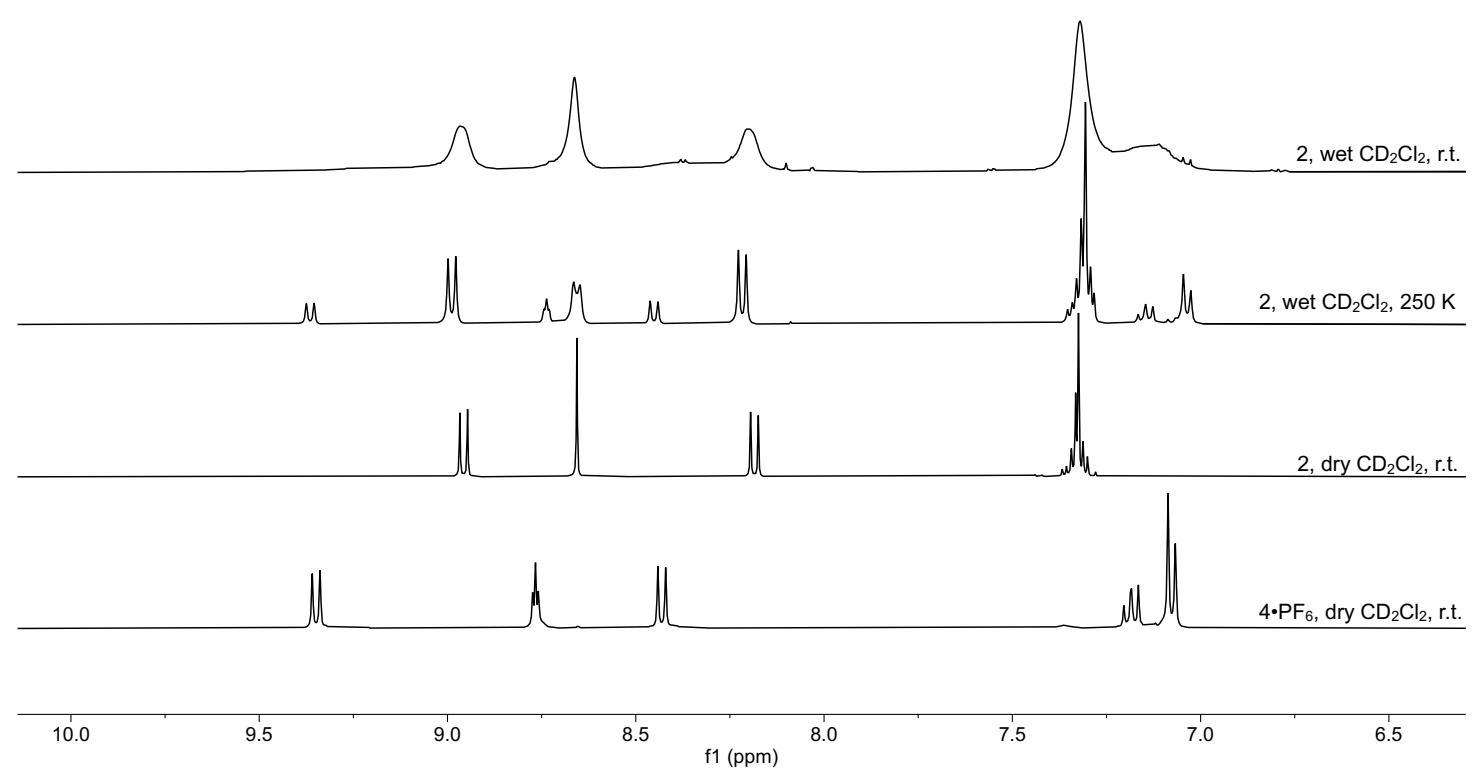

Figure S22. Variable-temperature ${ }^{1} \mathrm{H}$ NMR spectra for compound 2 after exposure to humid ambient atmosphere: at room temperature, the signals for the iPrNDI ligand are substantially broadened; upon cooling, the spectrum resolves into two sets of signals corresponding to compounds 2 and $\mathbf{4}$. Analogous behavior is observed when a dry $\mathrm{CD}_{2} \mathrm{Cl}_{2}$ solution of $\mathbf{2}$ is intentionally spiked with small amounts of either $\mathrm{D}_{2} \mathrm{O}$ or $\mathrm{CD}_{3} \mathrm{CN}$.

\section{DFT Calculations}

Density functional theory (DFT) calculations were performed using the Gaussian 16 program package, ${ }^{9}$ and the GaussView 6 interface. ${ }^{10}$ Geometry optimizations were carried out using the atomic coordinates from the crystal structures of 2, 3, and $\mathbf{4}$ as starting points; for computational convenience, the $\operatorname{Pr}$ groups of the ligands were replaced with methyl groups. Calculations were performed using the B3PW91 functional and the LanL2DZ basis set. Frequency calculations on the optimized geometries using the same method resulted in no imaginary frequencies, confirming that the geometries are indeed local minima.

Time-dependent DFT (TD-DFT) calculations were performed on the geometry-optimized structures, using B3PW91/LanL2DZ and the polarizable continuum model with dichloromethane as the solvent. Analysis of simulated UV-vis spectra, molecular orbitals, and atomic contributions to the frontier molecular orbitals (Mulliken contribution) were carried out using Chemissian. ${ }^{11}$ Images were generated using GaussView 6, Chemissian, or CrystalMaker. All molecular orbital visualizations were generated using an isosurface value of 0.03 . 
Table S5. Cartesian Coordinates for the Optimized Structure of 1

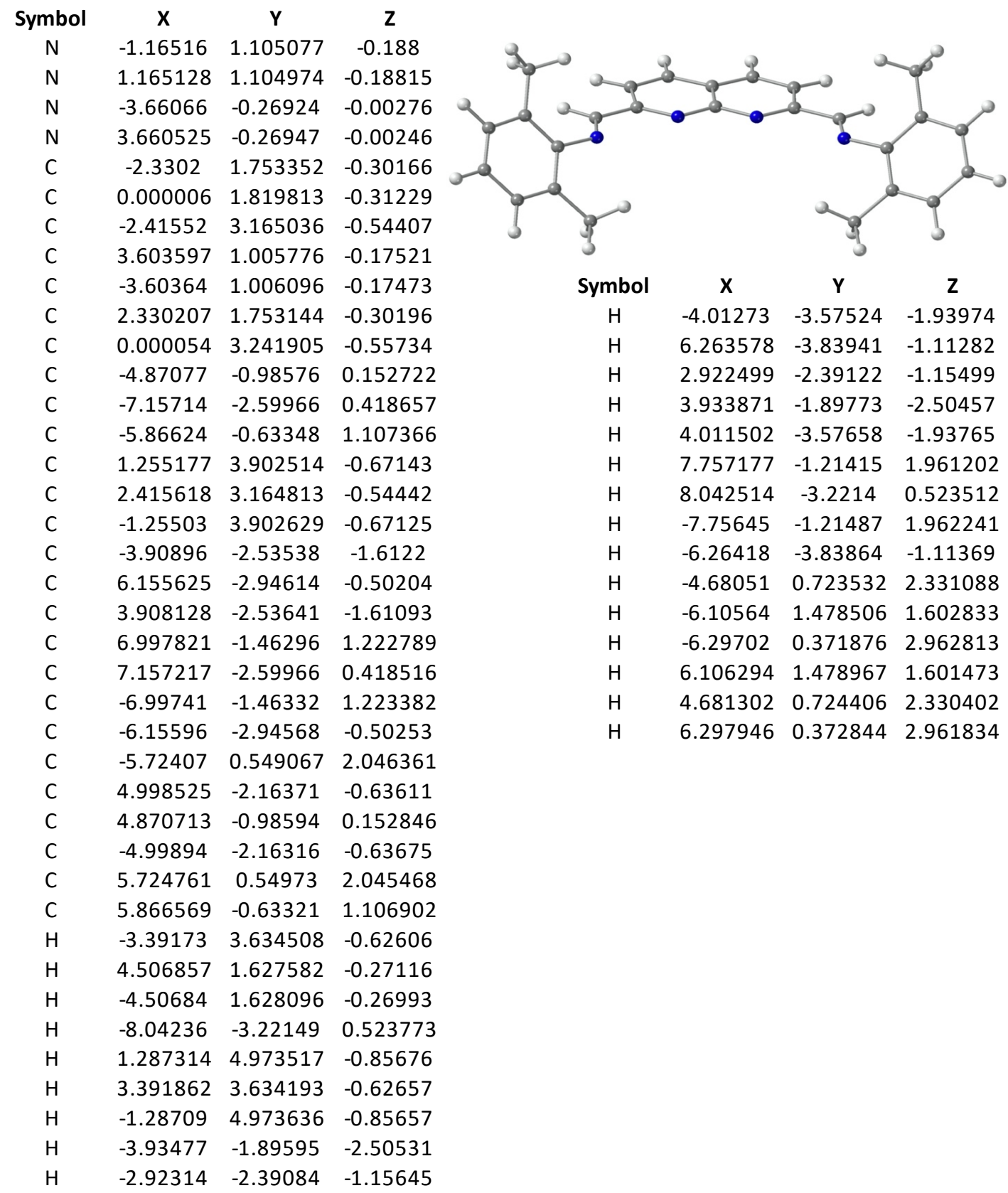


Table S6. Selected Frontier MOs for 1

\begin{tabular}{|c|c|c|}
\hline $\begin{array}{c}\text { MO \# } \\
\text { (Energy, eV) }\end{array}$ & Fragment Analysis & $\begin{array}{c}\text { Visualization } \\
\text { (Isosurface value } 0.03 \text { ) }\end{array}$ \\
\hline $\begin{array}{c}\text { 107, LUMO+2 } \\
(-0.870)\end{array}$ & $\begin{array}{c}\text { naphthyridine }(37 \%) \\
\text { imines }(26 \%) \\
\text { aryl rings }(35 \%)\end{array}$ & \\
\hline $\begin{array}{l}\text { 106, LUMO+1 } \\
(-2.257)\end{array}$ & $\begin{array}{c}\text { naphthyridine }(52 \%) \\
\text { imines }(31 \%) \\
\text { aryl rings }(17 \%)\end{array}$ & \\
\hline $\begin{array}{c}\text { 105, LUMO } \\
(-3.000)\end{array}$ & $\begin{array}{c}\text { naphthyridine }(64 \%) \\
\text { imines }(24 \%) \\
\text { aryl rings }(11 \%)\end{array}$ & \\
\hline $\begin{array}{c}\text { 104, HOMO } \\
(-6.186)\end{array}$ & $\begin{array}{c}\text { aryl rings }(55 \%) \\
\text { imines }(31 \%) \\
\text { naphthyridine }(9 \%)\end{array}$ & \\
\hline
\end{tabular}




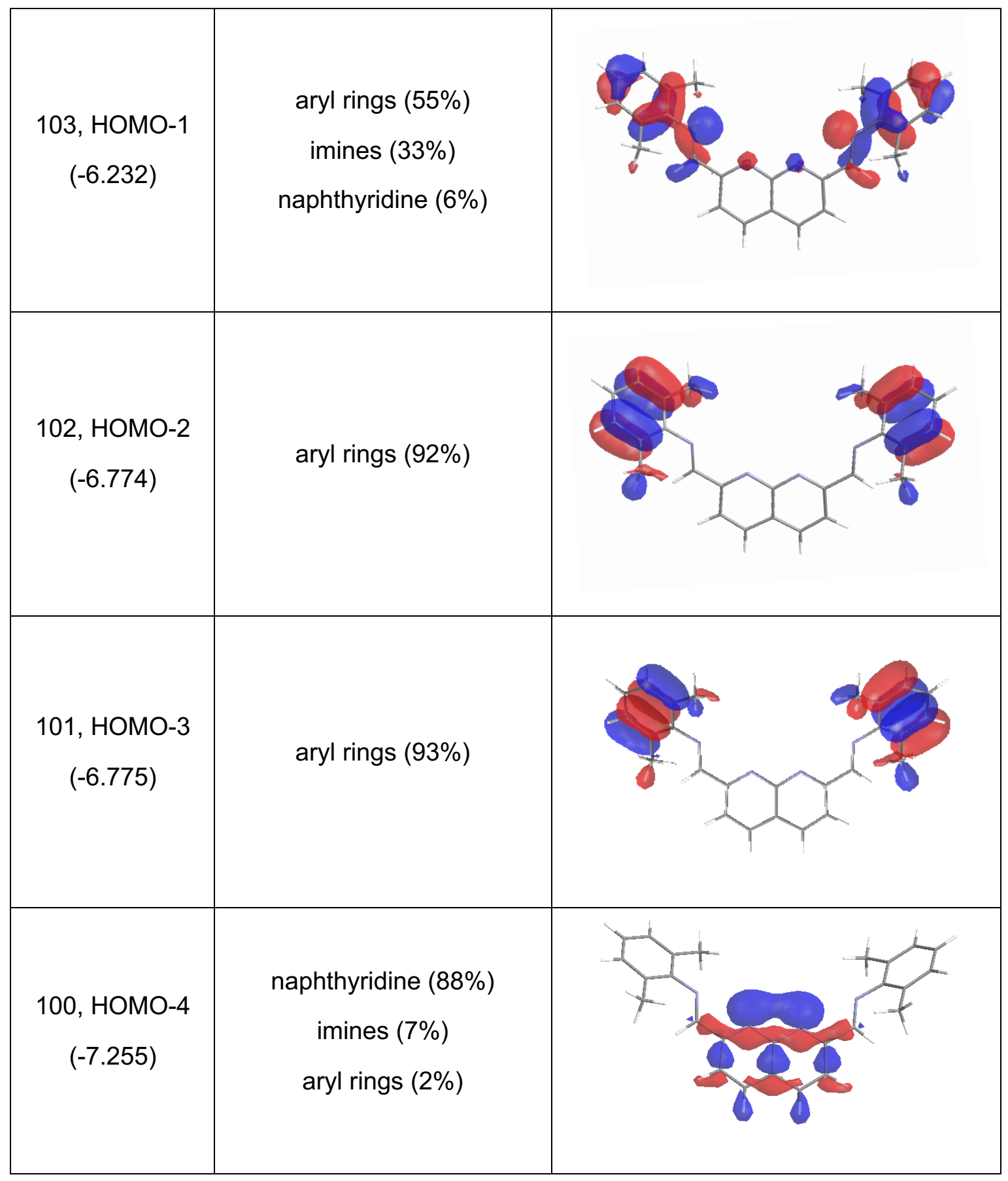


Table S7. Cartesian Coordinates for the Optimized Structure of 2

\begin{tabular}{|c|c|c|c|c|c|c|c|}
\hline Atom & X & $\mathbf{Y}$ & Z & & & & \\
\hline $\mathrm{Ag}$ & 12.115419 & 7.716602 & 6.458607 & & & & \\
\hline $\mathrm{Ag}$ & 9.12962 & 7.718838 & 6.455514 & & & & \\
\hline$S$ & 10.61992 & 5.833284 & 9.10294 & & & & \\
\hline$S$ & 10.624545 & 10.813757 & 5.96965 & & & & \\
\hline $\mathrm{F}$ & 10.618 & 3.472051 & 7.609696 & & & & \\
\hline $\mathrm{F}$ & 9.500304 & 13.022367 & 7.215301 & & & & \\
\hline$F$ & 9.493339 & 3.304107 & 9.544707 & & & & \\
\hline $\mathrm{F}$ & 11.738767 & 3.301062 & 9.546736 & & & & \\
\hline $\mathrm{F}$ & 10.617638 & 11.660598 & 8.608855 & & & & \\
\hline $\mathrm{F}$ & 11.745286 & 13.020497 & 7.221784 & Atom & $x$ & $\mathbf{Y}$ & $\mathbf{Z}$ \\
\hline 0 & 11.979911 & 9.960562 & 6.353203 & $\mathrm{H}$ & 15.008125 & 8.929947 & 3.936821 \\
\hline 0 & 9.263321 & 6.211258 & 8.257569 & $\mathrm{H}$ & 14.102359 & 9.718472 & .233661 \\
\hline 0 & 9.265669 & 9.962663 & 6.34546 & $\mathrm{H}$ & 15.60 & 10.444745 & 636548 \\
\hline 0 & 10.629213 & 11.552 & 4.5 & C & 4.159 & 9.259241 & 267 \\
\hline 0 & 10.619766 & 6.155881 & 10.679062 & $\mathrm{H}$ & 3.809 & 10.229488 & 110955 \\
\hline 0 & 11.978136 & 6.207569 & $8.25 \varepsilon$ & C & 10.61 & 3.840022 & .951364 \\
\hline $\mathrm{N}$ & 11.793588 & 5.815404 & 4.984125 & C & 6.13058 & 9.495 & 4.869551 \\
\hline $\mathrm{N}$ & 9.450966 & 5.815 & 4.984198 & $\mathrm{H}$ & 7.143438 & 9.718995 & 5.230405 \\
\hline $\mathrm{N}$ & 14.255366 & 6.936632 & 5.819217 & $\mathrm{H}$ & 6.236566 & 8.930503 & 3.93432 \\
\hline $\mathrm{N}$ & 6.989377 & 6.937942 & 5.817819 & $\mathrm{H}$ & 5.642323 & 10.445925 & 4.633785 \\
\hline C & 12.961187 & 5.243447 & 4.65597 & C & 3.915291 & 7.324161 & 7.900864 \\
\hline C & 10.622255 & 5.227985 & 4.587056 & $\mathrm{H}$ & 3.383556 & 6.804404 & 8.694052 \\
\hline C & 13.038467 & 4.051714 & 3.873206 & C & 3.4464 & 8.562876 & 7.440521 \\
\hline $\mathrm{H}$ & 14.011509 & 3.636067 & 3.631657 & $\mathrm{H}$ & 2.54244 & 8.992591 & 7.863494 \\
\hline C & 7.020805 & 5.839967 & 5.142697 & C & 17.329835 & 7.320715 & 7.902016 \\
\hline $\mathrm{H}$ & 6.106773 & 5.288761 & 4.897906 & $\mathrm{H}$ & 17.86148 & 6.800275 & 8.694821 \\
\hline C & 14.223829 & 5.839841 & 5.1 & $C$ & 17.085891 & 9.256722 & 6.45558 \\
\hline $\mathrm{H}$ & 15.137898 & 5.289326 & 4.895922 & $\mathrm{H}$ & 17.436891 & 10.227051 & 6.113801 \\
\hline C & 8.283311 & 5.2433 & 4.65 & C & 15.631256 & 5.440773 & 7.942754 \\
\hline C & 10.622207 & 4.018724 & 3.805876 & $\mathrm{H}$ & 14.554823 & 5.502103 & 8.143881 \\
\hline C & 15.477557 & 7.4 & 6.3 & $\mathrm{H}$ & 15.799 & 4.58545 & 7.274986 \\
\hline C & 17.799071 & 8.559554 & 7.442384 & $\mathrm{H}$ & 16.133965 & 5.213001 & 8.887662 \\
\hline $\mathrm{H}$ & 18.7032 & 8.988743 & 7.86 & C & 10.621737 & 12.23602 & 7.360527 \\
\hline C & 16.16419 & 6.739211 & 7.37062 & C & 5.340983 & 8.731596 & 5.905322 \\
\hline C & 9.370982 & 3.4464 & 3.446922 & C & 5.767473 & 7.457174 & 6.354784 \\
\hline $\mathrm{H}$ & 9.345537 & 2.536345 & 2.853864 & C & 15.904432 & 8.729784 & 5.9074 \\
\hline C & 8.205945 & 4.051349 & 3.874068 & C & 5.613278 & 5.443778 & 7.942812 \\
\hline $\mathrm{H}$ & 7.232887 & 3.635491 & 3.632949 & $\mathrm{H}$ & 5.444392 & 4.58791 & 7.2759 \\
\hline C & 11.873371 & 3.446621 & 3.4464 & $\mathrm{H}$ & 6.6898 & 5.504955 & 8.143595 \\
\hline $\mathrm{H}$ & 11.898747 & 2.536635 & 2.853235 & $\mathrm{H}$ & 5.110758 & 5.217052 & 8.888068 \\
\hline C & 15.114901 & 9.494289 & 4.872203 & C & 5.080677 & 6.741934 & 7.369719 \\
\hline
\end{tabular}


Table S8. Selected Frontier MOs for 2

\begin{tabular}{|c|c|c|}
\hline $\begin{array}{c}\text { MO \# } \\
\text { (Energy, eV) }\end{array}$ & $\begin{array}{l}\text { Fragment } \\
\text { Analysis }\end{array}$ & $\begin{array}{c}\text { Visualization } \\
\text { (Isosurface value } 0.03 \text { ) }\end{array}$ \\
\hline $\begin{array}{c}\text { 190, LUMO+3 } \\
(-2.193)\end{array}$ & $\begin{array}{c}\text { OTf: } 96 \% \\
\text { naphthyridine: } 2 \% \\
\text { Ag: } 1 \%\end{array}$ & \\
\hline $\begin{array}{c}\text { 189, LUMO+2 } \\
(-2.558)\end{array}$ & $\begin{array}{c}\text { OTf: } 92 \% \\
\text { Ag: } 5 \% \\
\text { naphthyridine: } 1 \%\end{array}$ & \\
\hline $\begin{array}{c}\text { 188, LUMO+1 } \\
(-2.789)\end{array}$ & $\begin{array}{c}\text { naphthyridine: } 49 \% \\
\text { imines: } 34 \% \\
\text { aryl rings: } 9 \% \\
\text { OTf: } 6 \%\end{array}$ & \\
\hline
\end{tabular}




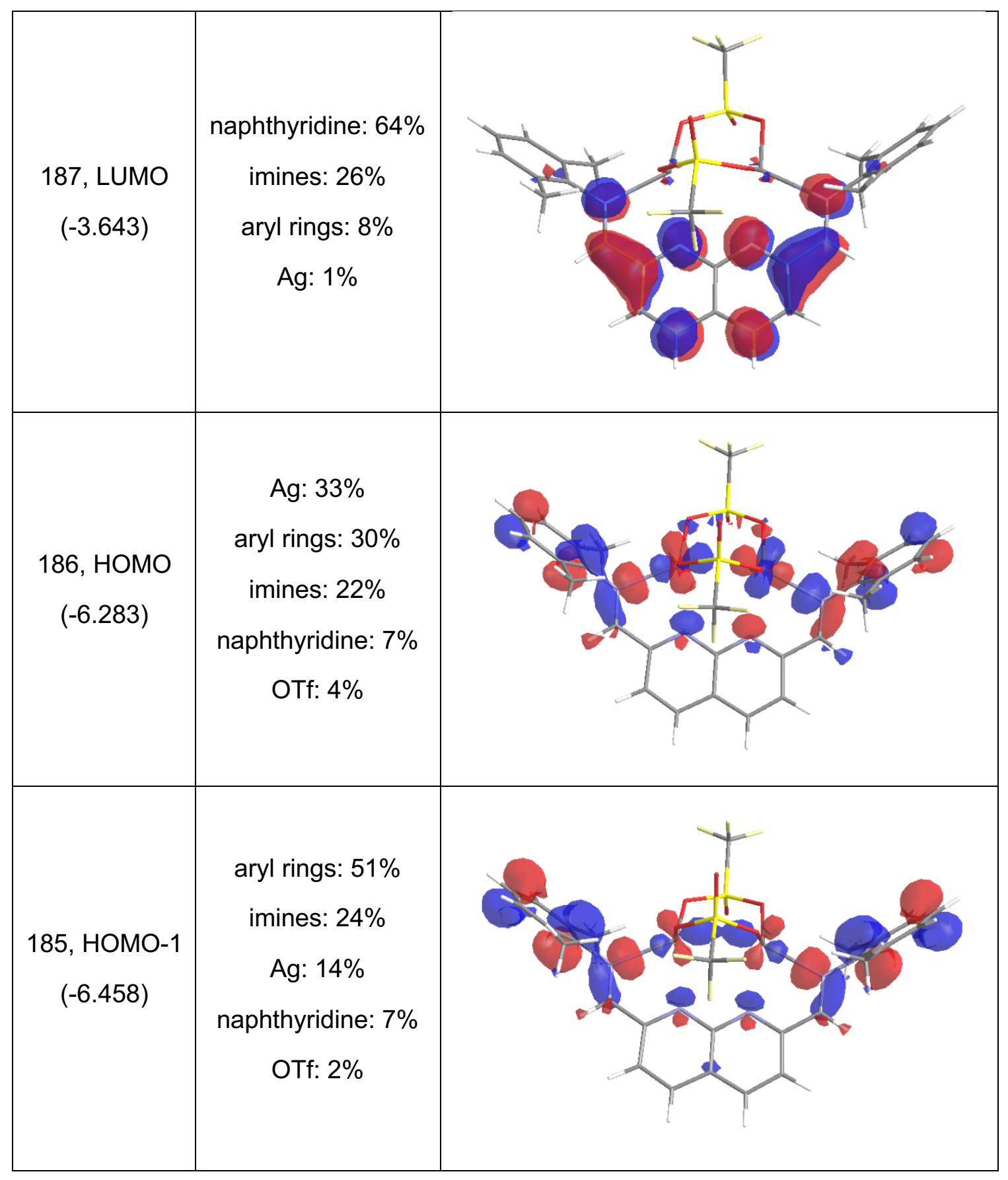




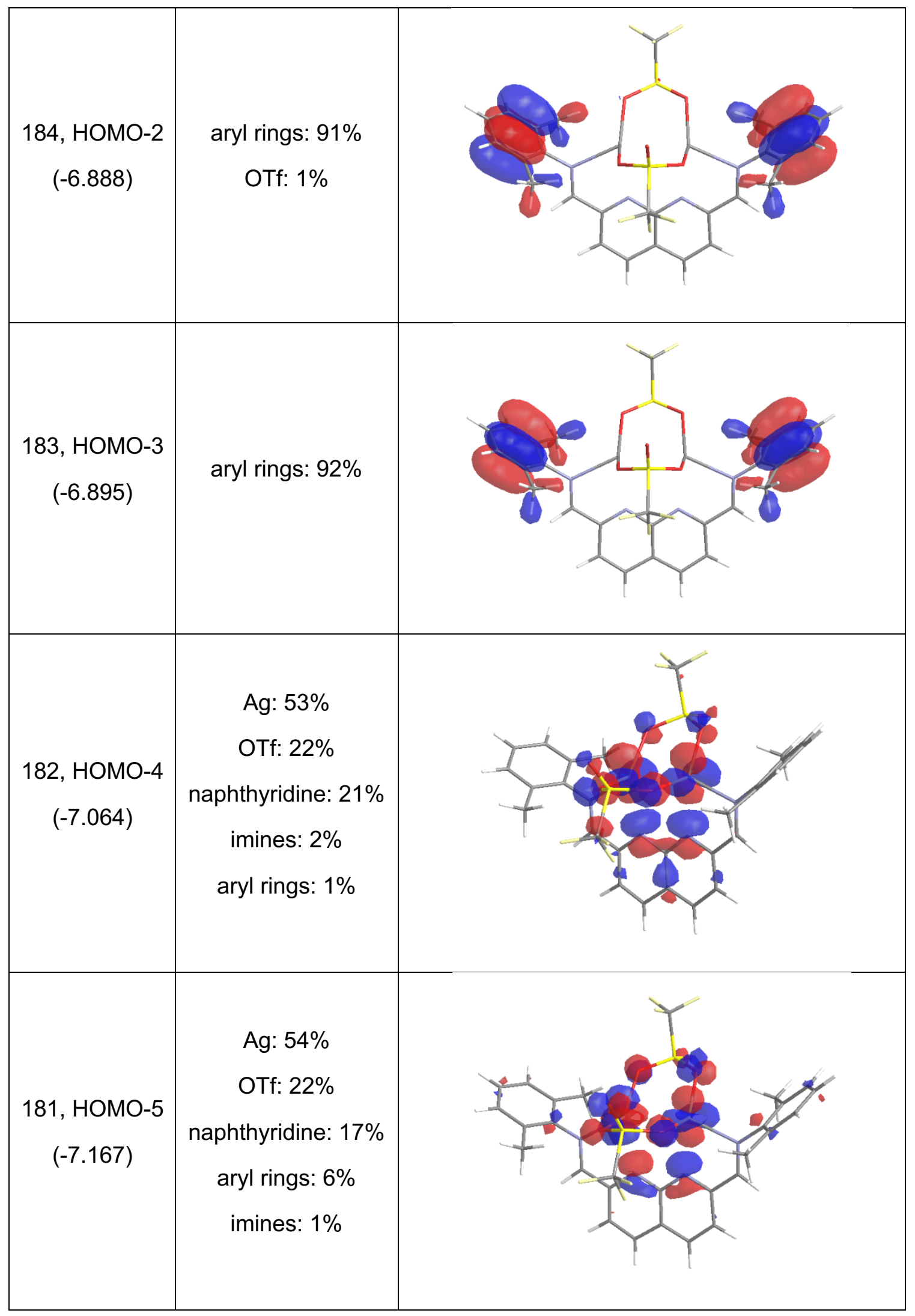




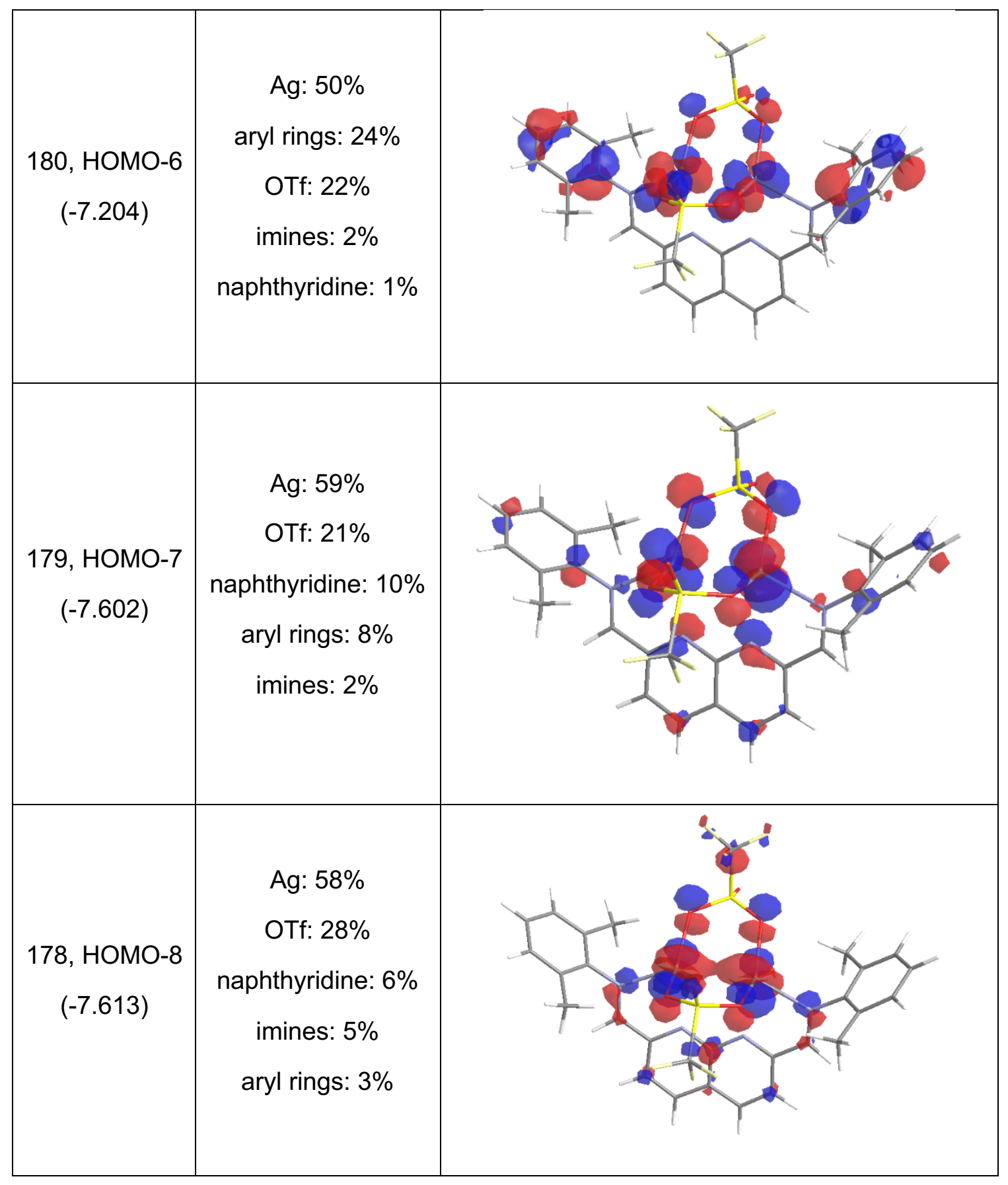




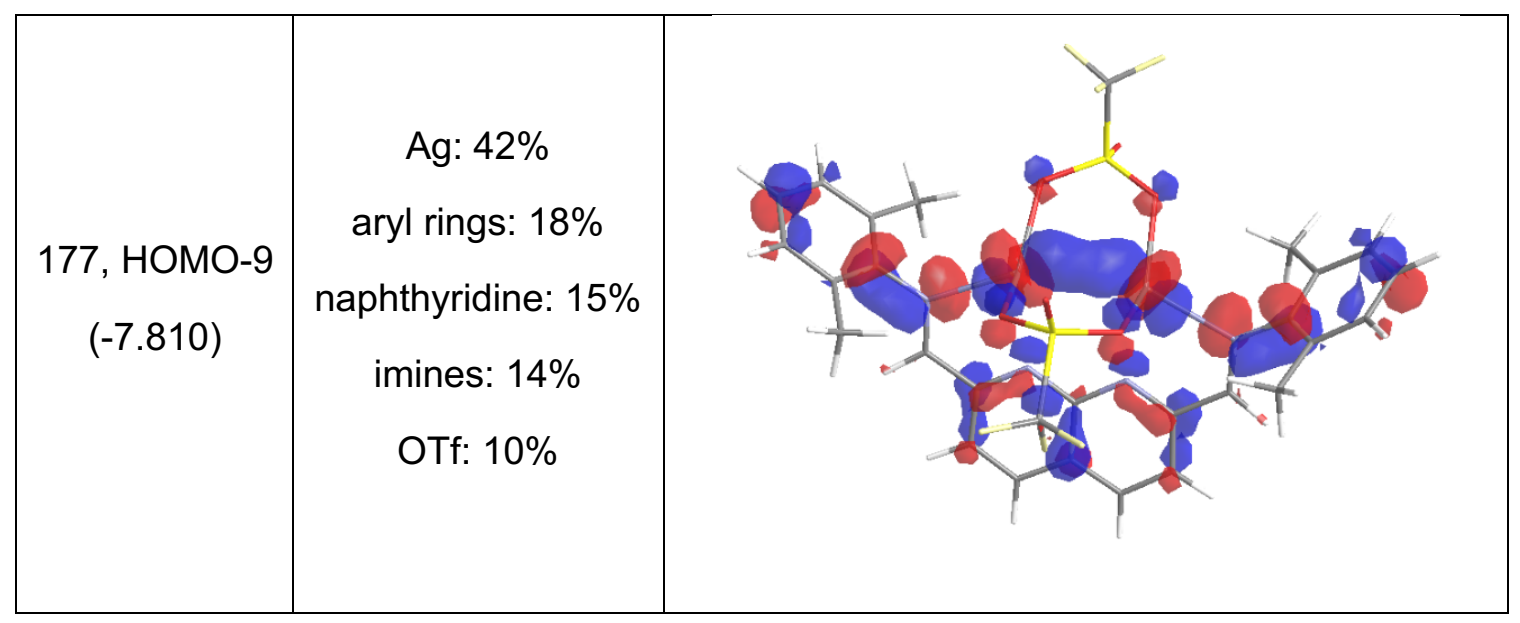

Table S9. Primary Visible-Region Transitions for 2, Predicted by TD-DFT

\begin{tabular}{|l|l|l|}
\hline Energy $(\mathbf{n m})$ & Oscillator Strength & $\begin{array}{l}\text { Major Contributors } \\
\text { (amplitude) }\end{array}$ \\
\hline 581 & 0.074 & $\begin{array}{l}185 \rightarrow 187(0.95) \\
186 \rightarrow 188(0.24)\end{array}$ \\
\hline 423 & 0.049 & $180 \rightarrow 187(0.90)$ \\
& & $185 \rightarrow 188(0.31)$ \\
& & $181 \rightarrow 187(0.27)$ \\
\hline 356 & 0.182 & $177 \rightarrow 187(0.90)$ \\
\hline
\end{tabular}

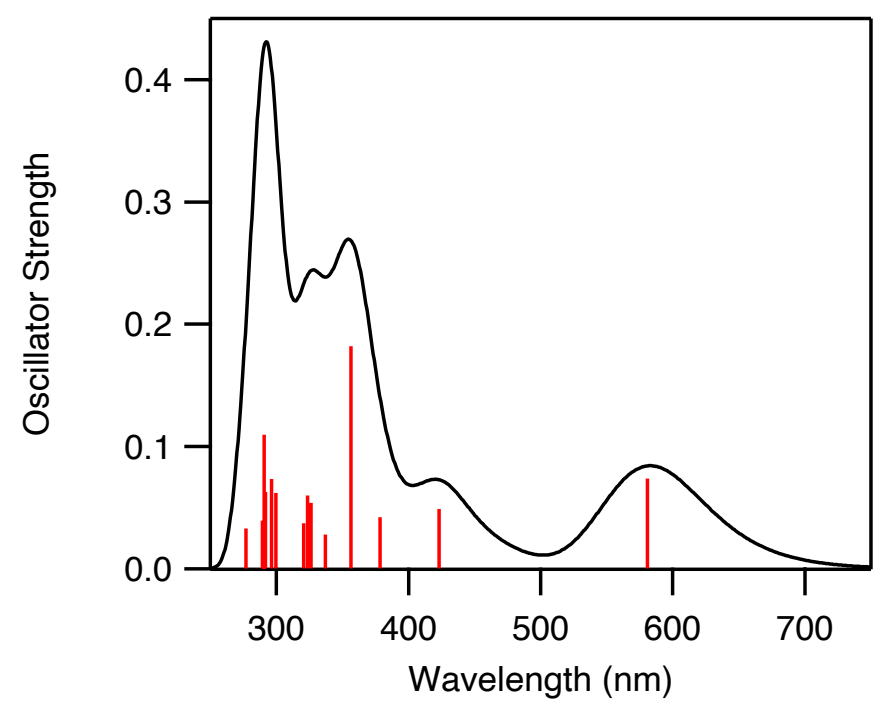

Figure S23. Simulated UV-vis spectrum for 2, from TD-DFT calculations (for clarity, only predicted transitions with an oscillator strength $\geq 0.03$ are shown). 
Table S10. Cartesian Coordinates for the Optimized Structure of 3

\begin{tabular}{|c|c|c|c|}
\hline Atom & $x$ & $\mathbf{Y}$ & Z \\
\hline $\mathrm{Ag}$ & -1.45296 & 0.045582 & -0.01824 \\
\hline $\mathrm{N}$ & 3.492098 & -4.00092 & -1.04468 \\
\hline$N$ & 3.655287 & 3.837033 & 1.143989 \\
\hline$N$ & -2.82237 & 1.602438 & -1.38446 \\
\hline$N$ & -0.79969 & 0.116387 & 2.156986 \\
\hline$N$ & 0.920964 & 1.57985 & 1.598242 \\
\hline $\mathrm{N}$ & -0.77829 & -0.10491 & -2.18598 \\
\hline$N$ & 0.842882 & -1.66277 & -1.58577 \\
\hline$N$ & -2.95703 & -1.4267 & 1.314729 \\
\hline C & 1.881066 & -2.44809 & -1.91502 \\
\hline C & -1.47409 & -0.62946 & 3.04616 \\
\hline C & 0.296893 & 0.832997 & 2.558849 \\
\hline C & 2.46445 & -3.24661 & -0.82347 \\
\hline $\mathrm{H}$ & 1.985474 & -3.10966 & 0.150214 \\
\hline C & -2.65359 & -1.37734 & 2.569436 \\
\hline $\mathrm{H}$ & -3.24113 & -1.89671 & 3.333098 \\
\hline C & -4.13108 & -2.09403 & 0.844839 \\
\hline C & -1.08958 & -0.71039 & 4.414263 \\
\hline $\mathrm{H}$ & -1.66763 & -1.3251 & 5.097049 \\
\hline C & 0.74616 & 0.794148 & 3.923364 \\
\hline C & -5.42384 & -1.67393 & 1.257703 \\
\hline C & -2.27517 & 3.735404 & 0.423334 \\
\hline $\mathrm{H}$ & -1.70623 & 2.837312 & 0.69336 \\
\hline $\mathrm{H}$ & -1.72349 & 4.220084 & -0.39351 \\
\hline $\mathrm{H}$ & -2.2731 & 4.420551 & 1.276937 \\
\hline C & -3.94285 & 2.365805 & -0.93052 \\
\hline C & 0.272668 & -0.89786 & -2.56561 \\
\hline C & 4.357568 & 4.607798 & 0.189471 \\
\hline C & 0.020296 & -0.00312 & 4.847906 \\
\hline $\mathrm{H}$ & 0.338536 & -0.04951 & 5.885855 \\
\hline C & -5.26259 & 2.047193 & -1.34905 \\
\hline C & -3.68711 & 3.399315 & 0.008008 \\
\hline C & 1.999295 & 2.297643 & 1.951489 \\
\hline C & -1.39483 & 0.668663 & -3.09319 \\
\hline C & 2.633863 & 3.087369 & 0.882361 \\
\hline $\mathrm{H}$ & 2.15072 & 3.00561 & -0.09553 \\
\hline C & 2.410772 & -2.52398 & -3.24664 \\
\hline $\mathrm{H}$ & 3.249051 & -3.18804 & -3.42114 \\
\hline C & 4.785823 & 5.891906 & 0.63667 \\
\hline C & 3.386958 & -5.63767 & 0.847574 \\
\hline C & 1.897099 & 1.568223 & 4.263849 \\
\hline $\mathrm{H}$ & 2.265418 & 1.560684 & 5.286259 \\
\hline C & 4.710729 & 4.130534 & -1.10723 \\
\hline C & -3.95289 & -3.13465 & -0.10333 \\
\hline C & 4.434901 & 6.375624 & 2.022991 \\
\hline $\mathrm{H}$ & 3.359126 & 6.570283 & 2.121085 \\
\hline $\mathrm{H}$ & 4.688079 & 5.618846 & 2.773894 \\
\hline
\end{tabular}

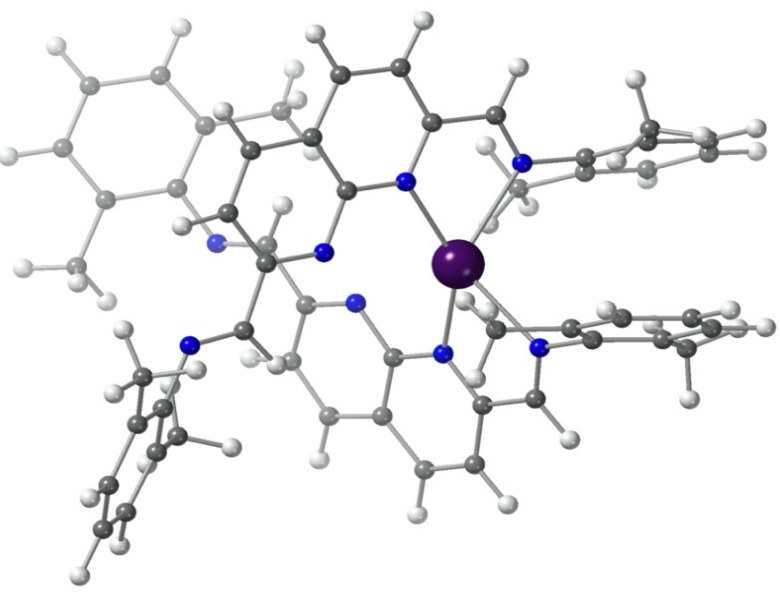

$\begin{array}{cccc}\text { Atom } & \mathbf{X} & \mathbf{Y} & \mathbf{Z} \\ \text { H } & 4.967875 & 7.301889 & 2.258968 \\ \mathrm{C} & -6.38064 & -3.38431 & -0.21172 \\ \mathrm{H} & -7.25437 & -3.88643 & -0.61732 \\ \mathrm{C} & 5.531838 & -4.89088 & -0.14824 \\ \mathrm{C} & -5.09432 & -3.77552 & -0.61249 \\ \mathrm{H} & -4.97124 & -4.58662 & -1.32569 \\ \mathrm{C} & -5.5695 & 0.907171 & -2.29986 \\ \mathrm{H} & -5.44456 & 1.200222 & -3.35047 \\ \mathrm{H} & -4.93221 & 0.034307 & -2.11946 \\ \mathrm{H} & -6.60889 & 0.587073 & -2.18061 \\ \mathrm{C} & 5.511335 & 6.708677 & -0.24286 \\ \mathrm{H} & 5.824443 & 7.695979 & 0.086201 \\ \mathrm{C} & 0.732843 & -0.91373 & -3.92701 \\ \mathrm{C} & 0.064406 & -0.09082 & -4.87236 \\ \mathrm{H} & 0.390466 & -0.08622 & -5.90893 \\ \mathrm{C} & 1.837289 & -1.76254 & -4.2427 \\ \mathrm{H} & 2.211738 & -1.79871 & -5.26231 \\ \mathrm{C} & -2.56937 & -3.57649 & -0.5154 \\ \mathrm{H} & -1.93066 & -2.72237 & -0.77161 \\ \mathrm{H} & -2.06257 & -4.11201 & 0.298852 \\ \mathrm{H} & -2.61628 & -4.2499 & -1.37707 \\ \mathrm{C} & 6.219349 & -5.71261 & 0.756736 \\ \mathrm{H} & 7.30497 & -5.74874 & 0.72167 \\ \mathrm{C} & 6.285899 & -4.05891 & -1.15743 \\ \mathrm{H} & 6.22042 & -2.98795 & -0.92599 \\ \mathrm{H} & 7.344555 & -4.33569 & -1.17718 \\ \mathrm{H} & 5.86557 & -4.19097 & -2.16073 \\ \mathrm{C} & 5.849334 & 6.259103 & -1.52847 \\ \mathrm{H} & 6.425031 & 6.895119 & -2.19507 \\ \mathrm{C} & 5.465218 & 4.976134 & -1.94094 \\ \mathrm{H} & 5.760566 & 4.617006 & -2.92401 \\ \mathrm{C} & 1.873222 & -5.70371 & 0.89733\end{array}$


Table S10, ctd.

$\begin{array}{cccc}\text { Atom } & \mathbf{X} & \mathbf{Y} & \mathbf{Z} \\ \mathrm{H} & 1.448566 & -4.93348 & 1.555053 \\ \mathrm{H} & 1.418108 & -5.57946 & -0.09193 \\ \mathrm{H} & 1.55498 & -6.67431 & 1.29115 \\ \mathrm{C} & 5.522225 & -6.4974 & 1.688106 \\ \mathrm{H} & 6.065258 & -7.14256 & 2.373084 \\ \mathrm{C} & 4.380531 & 2.736348 & -1.60284 \\ \mathrm{H} & 4.384974 & 1.994886 & -0.79575 \\ \mathrm{H} & 3.39428 & 2.693164 & -2.08413 \\ \mathrm{H} & 5.117454 & 2.422074 & -2.34909 \\ \mathrm{C} & 2.52275 & 2.313887 & 3.287552 \\ \mathrm{H} & 3.399994 & 2.919968 & 3.480431 \\ \mathrm{C} & -6.53637 & -2.33662 & 0.70637 \\ \mathrm{H} & -7.53421 & -2.02195 & 1.001929 \\ \mathrm{C} & 4.109026 & -4.83078 & -0.08088 \\ \mathrm{C} & -4.77659 & 4.134774 & 0.502809 \\ \mathrm{H} & -4.59301 & 4.941327 & 1.20807 \\ \mathrm{C} & -6.32162 & 2.802421 & -0.81147 \\ \mathrm{H} & -7.33971 & 2.566029 & -1.11097 \\ \mathrm{C} & 4.121472 & -6.46801 & 1.713891 \\ \mathrm{H} & 3.583445 & -7.1057 & 2.411312 \\ \mathrm{C} & -2.51946 & 1.508072 & -2.63683 \\ \mathrm{H} & -3.06337 & 2.056514 & -3.41218 \\ \mathrm{C} & -6.08776 & 3.843424 & 0.097543 \\ \mathrm{H} & -6.9208 & 4.41807 & 0.492354 \\ \mathrm{C} & -0.99828 & 0.69702 & -4.46006 \\ \mathrm{H} & -1.52966 & 1.336075 & -5.15821 \\ \mathrm{C} & -5.64328 & -0.52016 & 2.21601 \\ \mathrm{H} & -5.53865 & -0.82733 & 3.264742 \\ \mathrm{H} & -4.94225 & 0.303125 & 2.038593 \\ \mathrm{H} & -6.6559 & -0.12232 & 2.100703\end{array}$


Table S11. Selected Frontier MOs for 3

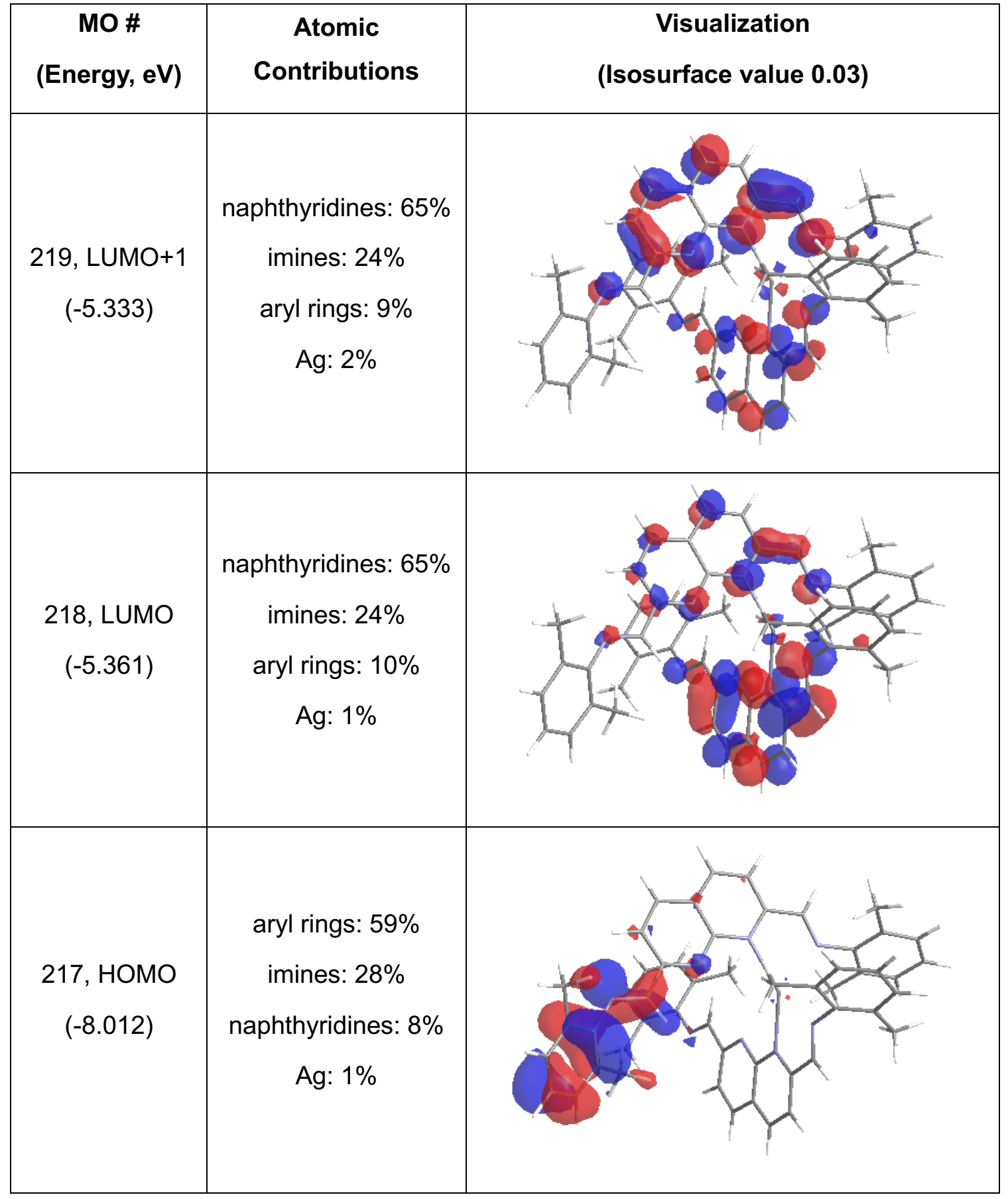




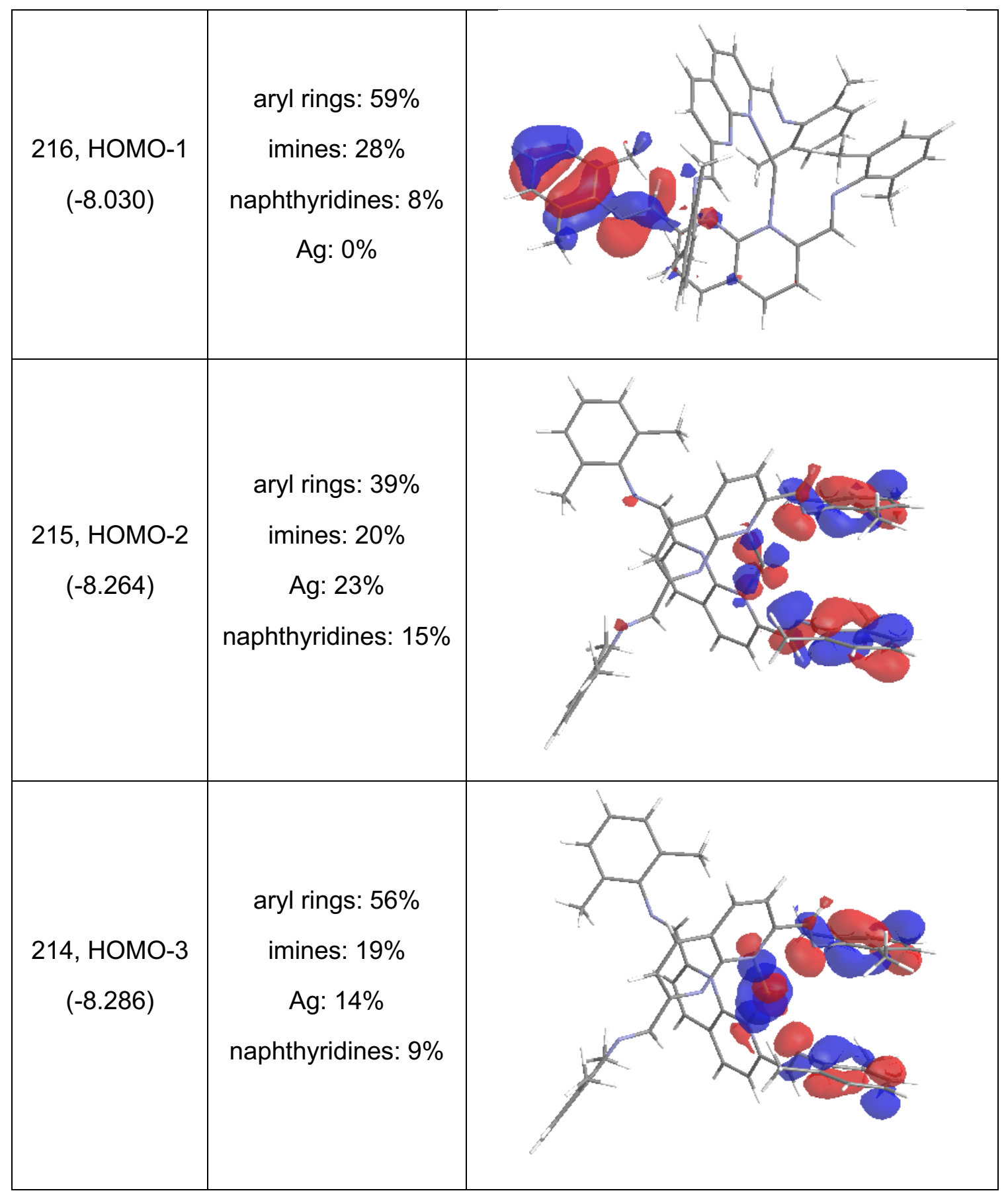




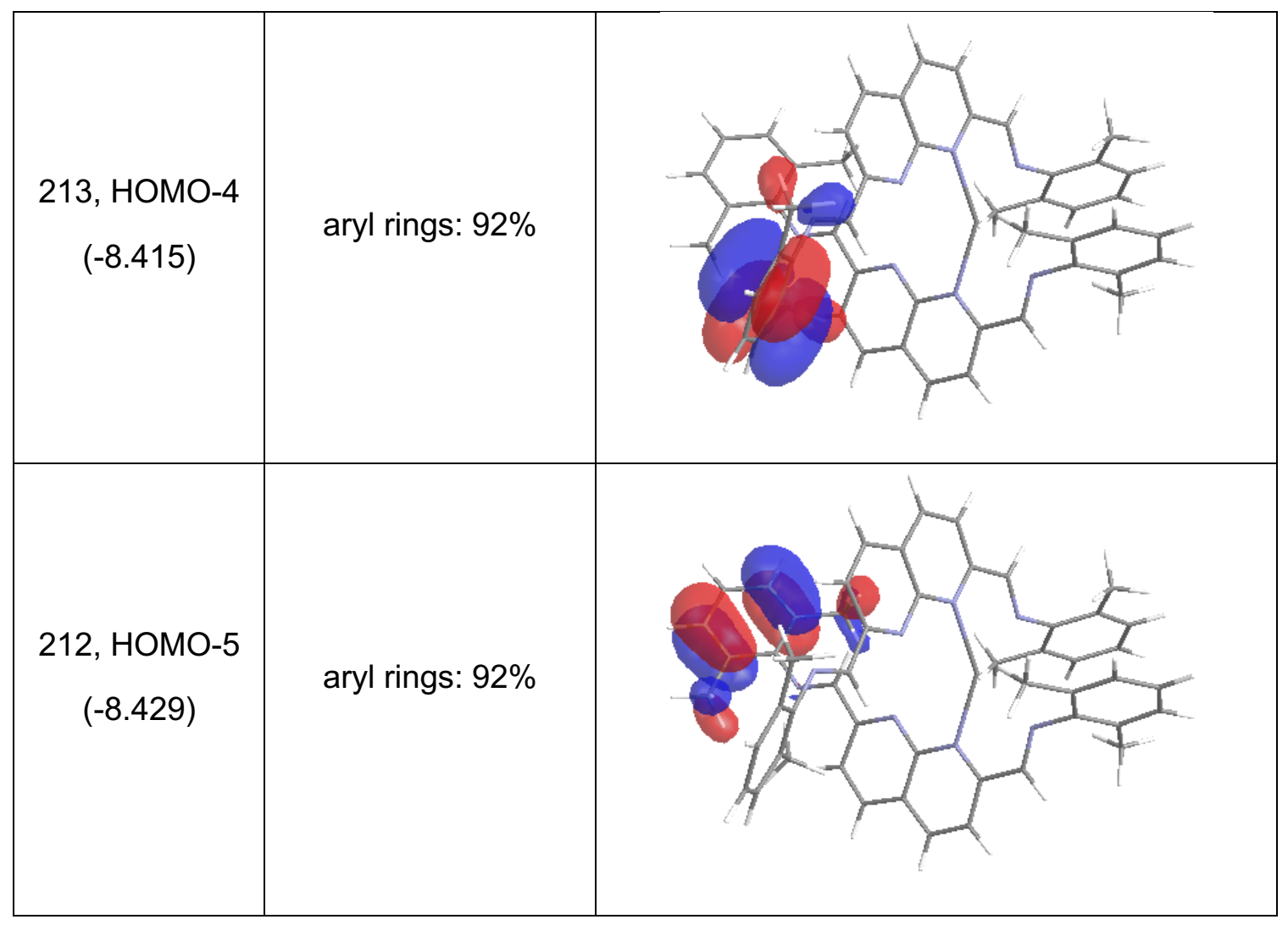


Table S12. Cartesian Coordinates for the Optimized Structure of 4

\begin{tabular}{|c|c|c|c|c|c|c|c|}
\hline Atom & X & $Y$ & z & & & & \\
\hline $\mathrm{Ag}$ & 11.485257 & 8.441482 & 7.041007 & & & & \\
\hline $\mathrm{Ag}$ & 8.462251 & 8.441484 & 7.040633 & & & & \\
\hline $\mathrm{N}$ & 11.002365 & 10.616904 & 7.598058 & & & & \\
\hline $\mathrm{N}$ & 11.002788 & 6.266128 & 6.483508 & & & & \\
\hline $\mathrm{N}$ & 8.946011 & 10.617984 & 6.487043 & & & & \\
\hline $\mathrm{N}$ & 8.946494 & 6.265078 & 7.594584 & & & & \\
\hline $\mathrm{N}$ & 13.11899 & 7.685364 & 5.305117 & & & & \\
\hline $\mathrm{N}$ & 13.11862 & 9.197773 & 8.776789 & & & & \\
\hline$N$ & 6.829916 & 7.68202 & 8.775085 & & & & \\
\hline $\mathrm{N}$ & 6.829669 & 9.201011 & 5.306345 & & & & \\
\hline C & 9.974194 & 11.330055 & 7.043201 & & & & \\
\hline C & 11.980701 & 5.61521 & 5.825836 & & & & \\
\hline C & 7.968855 & 5.612937 & 8.251463 & & & & \\
\hline C & 9.974241 & 12.76627 & 7.044494 & Atom & $x$ & $\mathbf{Y}$ & Z \\
\hline C & 8.928167 & 13.436667 & 6.350579 & C & 3.817326 & 10.094241 & 10.502995 \\
\hline $\mathrm{H}$ & 8.910468 & 14.522296 & 6.319019 & $\mathrm{H}$ & 3.055365 & 10.724178 & 10.952248 \\
\hline C & 7.968236 & 11.270147 & 5.830378 & C & 15.541913 & 8.779735 & 9.208011 \\
\hline C & 5.17427 & 10.42671 & 10.635398 & C & 15.542236 & 8.103423 & 4.873621 \\
\hline $\mathrm{H}$ & 5.461851 & 11.310023 & 11.19911 & C & 7.628204 & 9.966909 & 10.258928 \\
\hline C & 9.974743 & 5.552976 & 7.038567 & C & 13.776912 & 7.252241 & 10.05831 \\
\hline C & 11.980169 & 11.267839 & 8.25591 & C & 5.780685 & 8.449806 & 9.356646 \\
\hline C & 14.167983 & 8.454352 & 4.72486 & C & 8.929007 & 3.4464 & 7.731708 \\
\hline C & 13.0625 & 10.487379 & 8.880316 & $\mathrm{H}$ & 8.911429 & 2.360776 & 7.76351 \\
\hline $\mathrm{H}$ & 13.784083 & 11.059926 & 9.467175 & C & 6.171288 & 7.257423 & 4.021898 \\
\hline C & 6.885849 & 10.490766 & 5.204713 & C & 13.77716 & 9.63105 & 4.023732 \\
\hline $\mathrm{H}$ & 6.164289 & 11.064218 & 4.618736 & C & 16.502093 & 8.955123 & 4.296146 \\
\hline C & 12.001521 & 12.689794 & 8.36661 & $\mathrm{H}$ & 17.555107 & 8.710395 & 4.40826 \\
\hline $\mathrm{H}$ & 12.802511 & 13.168766 & 8.919924 & C & 7.947749 & 4.190814 & 8.35996 \\
\hline C & 11.020368 & 13.435364 & 7.739584 & $\mathrm{H}$ & 7.146872 & 3.71083 & 8.912559 \\
\hline $\mathrm{H}$ & 11.038143 & 14.520934 & 7.773084 & C & 3.817587 & 6.788032 & 3.578688 \\
\hline C & 14.167652 & 8.428862 & 9.356994 & $\mathrm{H}$ & 3.055788 & 6.157912 & 3.129416 \\
\hline C & 12.002255 & 4.193231 & 5.715459 & C & 11.021211 & 3.447671 & 6.342669 \\
\hline $\mathrm{H}$ & 12.803312 & 3.714241 & 5.162258 & $\mathrm{H}$ & 11.039145 & 2.362096 & 6.309431 \\
\hline C & 7.94697 & 12.692291 & 5.722195 & C & 4.406356 & 8.783691 & 4.874521 \\
\hline $\mathrm{H}$ & 7.146033 & 13.172313 & 5.169715 & C & 12.319749 & 6.910752 & 10.256753 \\
\hline C & 13.062925 & 6.39578 & 5.201355 & C & 12.319975 & 9.972674 & 3.825641 \\
\hline $\mathrm{H}$ & 13.784469 & 5.823344 & 4.614328 & C & 3.920461 & 9.98398 & 5.663236 \\
\hline C & 6.171139 & 9.625343 & 10.05986 & C & 4.406545 & 8.098795 & 9.207028 \\
\hline C & 6.886467 & 6.392324 & 8.877151 & C & 3.4464 & 8.949314 & 9.785728 \\
\hline $\mathrm{H}$ & 6.165221 & 5.818901 & 9.463558 & $\mathrm{H}$ & 2.393457 & 8.704567 & 9.673006 \\
\hline C & 9.974984 & 4.11677 & 7.037622 & C & 14.773724 & 10.433583 & 3.449348 \\
\hline C & 5.780595 & 8.432991 & 4.724989 & $\mathrm{H}$ & 14.485865 & 11.317801 & 2.887202 \\
\hline C & 5.174618 & 6.455811 & 3.4464 & C & 14.773541 & 6.449716 & 10.632593 \\
\hline $\mathrm{H}$ & 5.462387 & 5.572492 & 2.882798 & $\mathrm{H}$ & 14.485737 & 5.565561 & 11.194865 \\
\hline
\end{tabular}


Table S12, ctd.

$\begin{array}{cccc}\text { Atom } & \mathbf{X} & \mathbf{Y} & \mathbf{Z} \\ \mathrm{C} & 3.921014 & 6.898398 & 8.418264 \\ \mathrm{C} & 3.446403 & 7.932931 & 4.295845 \\ \mathrm{H} & 2.393409 & 8.177481 & 4.408525 \\ \mathrm{C} & 16.130785 & 10.101156 & 3.580883 \\ \text { H } & 16.8925 & 10.732002 & 3.132486 \\ \mathrm{C} & 7.628431 & 6.916085 & 3.822996 \\ \mathrm{C} & 16.028294 & 6.90187 & 5.660312 \\ \mathrm{C} & 16.027864 & 9.981227 & 8.421168 \\ \mathrm{C} & 16.501824 & 7.928045 & 9.785389 \\ \mathrm{H} & 17.554831 & 8.172719 & 9.673087 \\ \mathrm{C} & 16.130588 & 6.782074 & 10.500803 \\ \mathrm{H} & 16.892357 & 6.151233 & 10.949111 \\ \mathrm{H} & 8.115308 & 9.255069 & 10.937698 \\ \mathrm{H} & 8.185454 & 9.930881 & 9.313859 \\ \mathrm{H} & 7.735741 & 10.966264 & 10.691629 \\ \mathrm{H} & 12.211923 & 5.910846 & 10.688106 \\ \mathrm{H} & 11.832712 & 7.621811 & 10.936393 \\ \mathrm{H} & 11.762633 & 6.948181 & 9.311655 \\ \mathrm{H} & 11.832515 & 9.261301 & 3.146652 \\ \mathrm{H} & 11.763263 & 9.935984 & 4.771001 \\ \mathrm{H} & 12.212179 & 10.972344 & 3.39373 \\ \mathrm{H} & 3.826015 & 10.876724 & 5.031152 \\ \mathrm{H} & 2.924966 & 9.784489 & 6.070652 \\ \mathrm{H} & 4.574457 & 10.234695 & 6.505552 \\ \mathrm{H} & 4.574859 & 6.648241 & 7.575654 \\ \mathrm{H} & 3.827287 & 6.005459 & 9.050169 \\ \mathrm{H} & 2.925257 & 7.097412 & 8.01126 \\ \mathrm{H} & 8.115791 & 7.628568 & 3.145107 \\ \mathrm{H} & 8.185333 & 6.951179 & 4.768309 \\ \mathrm{H} & 7.736123 & 5.917131 & 3.389412 \\ \mathrm{H} & 15.374483 & 6.649818 & 6.502364 \\ \mathrm{H} & 16.122593 & 6.010122 & 5.026791 \\ \mathrm{H} & 17.02389 & 7.100704 & 6.067807 \\ \mathrm{H} & 16.12222 & 10.873019 & 9.054606 \\ \mathrm{H} & 17.023417 & 9.782384 & 8.013571 \\ \mathrm{H} & 15.373953 & 10.233201 & 7.579163\end{array}$


Table S13. Selected Frontier MOs for 4

\begin{tabular}{|c|c|c|}
\hline $\begin{array}{c}\text { MO \# } \\
\text { (Energy, eV) }\end{array}$ & Fragment Analysis & $\begin{array}{c}\text { Visualization } \\
\text { (Isosurface value } 0.03 \text { ) }\end{array}$ \\
\hline $\begin{array}{c}\text { 228, LUMO+1 } \\
(-4.192)\end{array}$ & $\begin{array}{c}\text { naphthyridines: } 65 \% \\
\text { imines: } 27 \% \\
\text { aryl rings: } 6 \% \\
\text { Ag: } 2 \%\end{array}$ & \\
\hline $\begin{array}{c}\text { 227, LUMO } \\
(-4.196)\end{array}$ & $\begin{array}{c}\text { naphthyridines: } 67 \% \\
\text { imines: } 25 \% \\
\text { aryl rings: } 7 \% \\
\text { Ag: } 1 \%\end{array}$ & \\
\hline $\begin{array}{c}226, \text { HOMO } \\
(-6.939)\end{array}$ & $\begin{array}{c}\text { aryl rings: } 54 \% \\
\text { imines: } 11 \% \\
\text { Ag: } 11 \% \\
\text { naphthyridines: } 6 \%\end{array}$ & \\
\hline
\end{tabular}




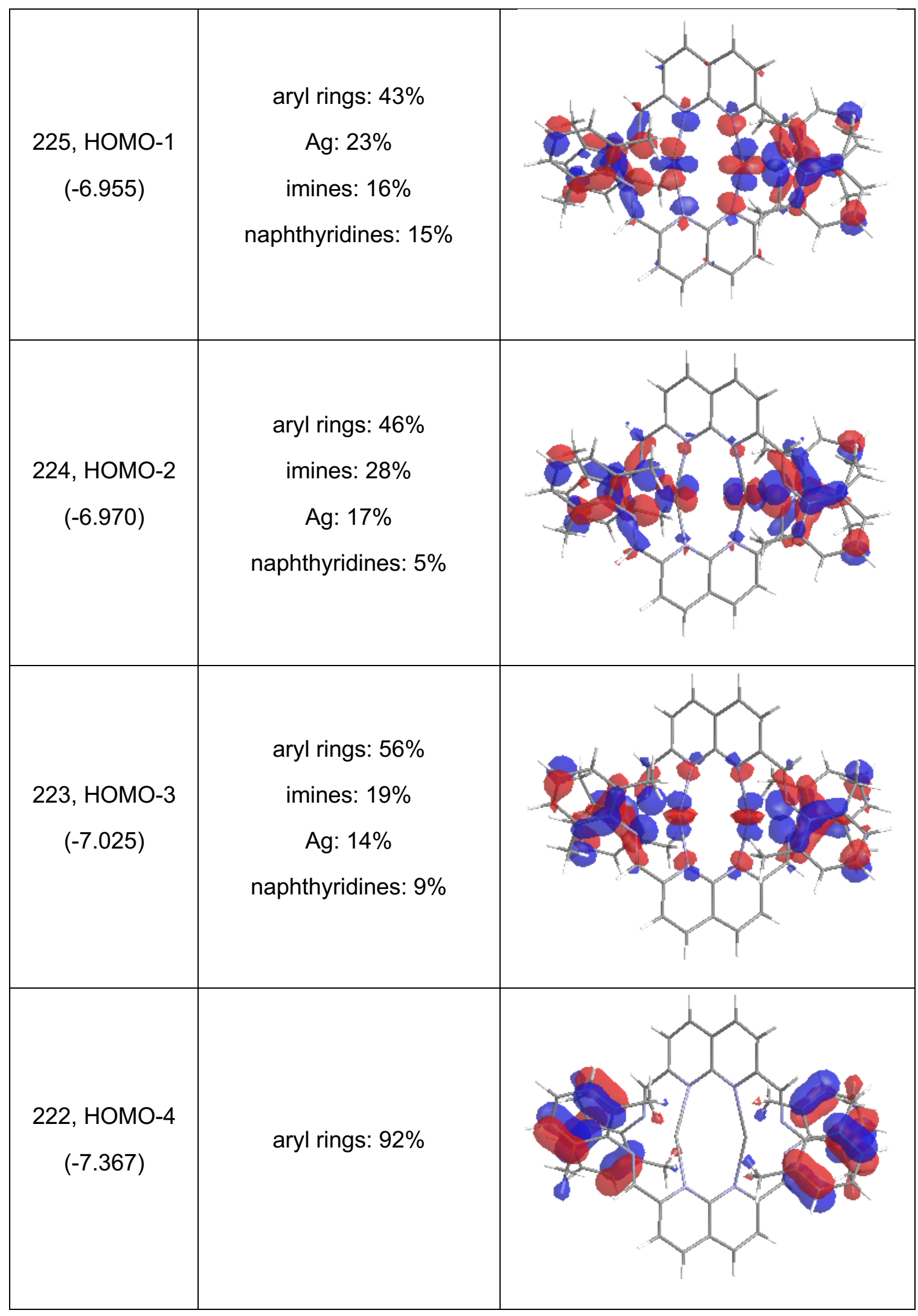




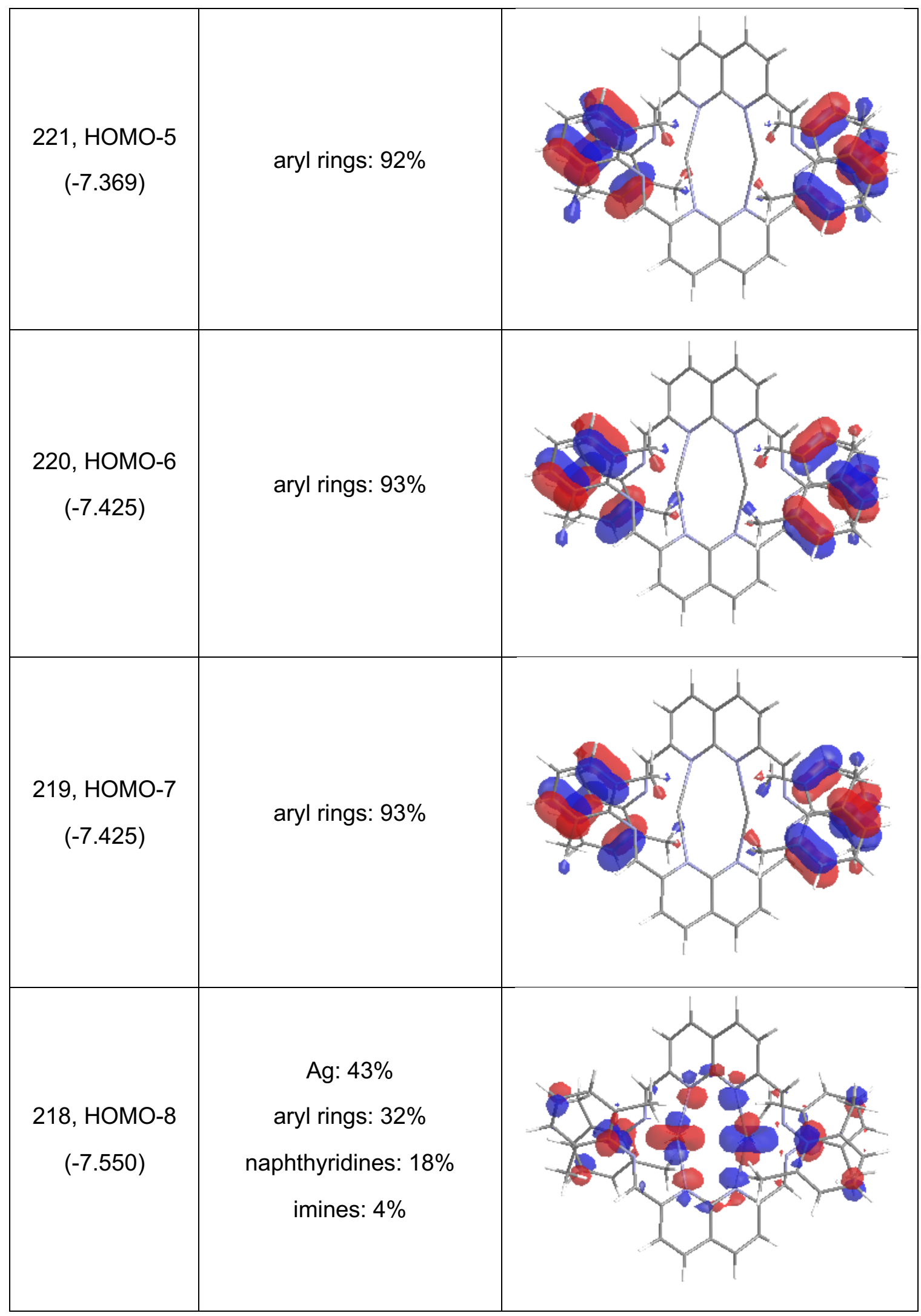




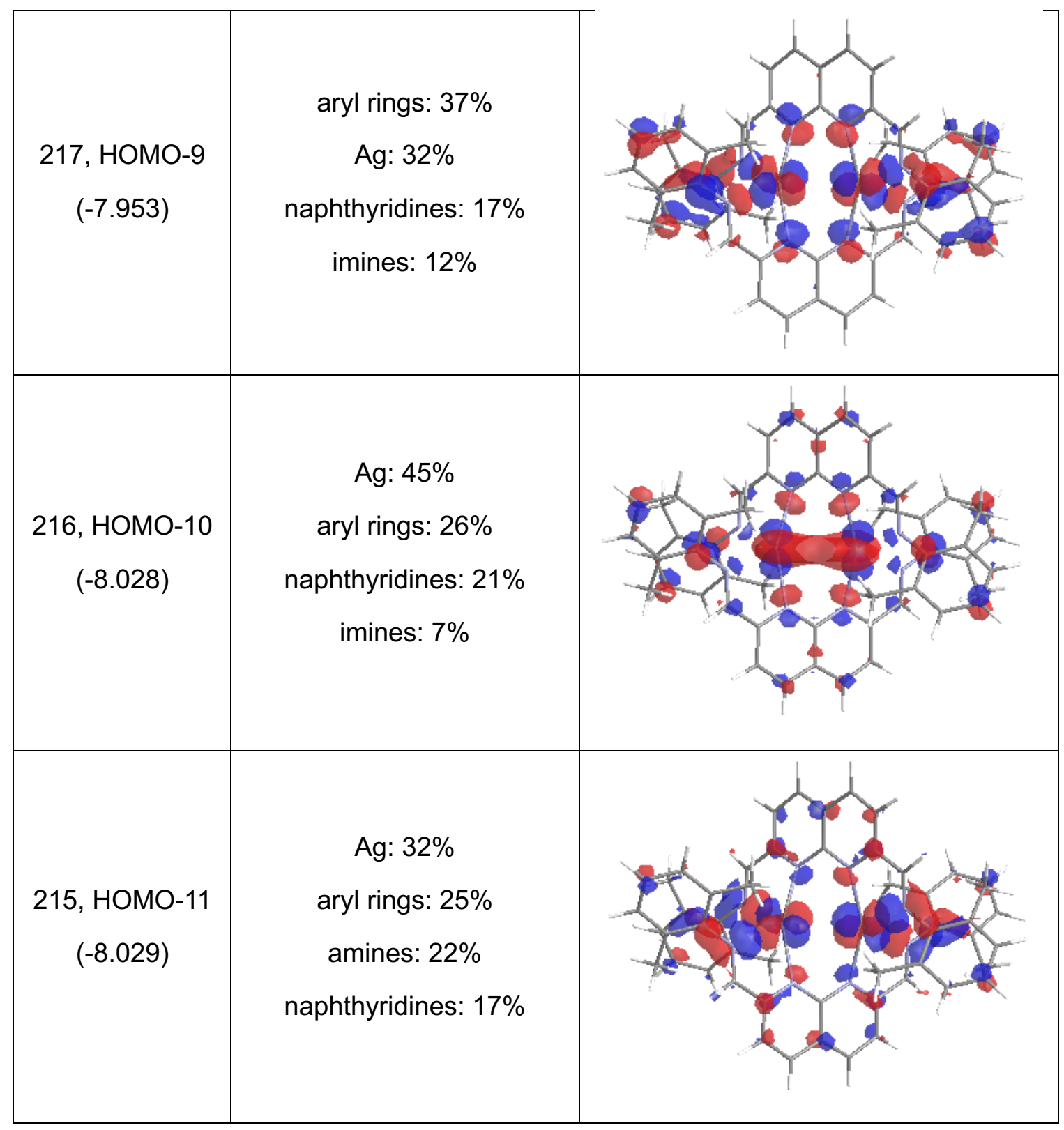


Table S14. Primary Visible-Region Transitions for 4, Predicted by TD-DFT

\begin{tabular}{|c|c|c|}
\hline Energy (nm) & Oscillator Strength & $\begin{array}{l}\text { Major Contributors } \\
\text { (amplitude) }\end{array}$ \\
\hline 597 & 0.136 & $\begin{array}{l}225 \rightarrow 228(0.83) \\
226 \rightarrow 227(0.48)\end{array}$ \\
\hline 453 & 0.189 & $218 \rightarrow 228(0.94)$ \\
\hline 384 & 0.104 & $216 \rightarrow 227(0.86)$ \\
\hline 382.8 & 0.113 & $\begin{array}{l}223 \rightarrow 230(0.71) \\
215 \rightarrow 228(0.66)\end{array}$ \\
\hline 382.7 & 0.301 & $215 \rightarrow 227(0.90)$ \\
\hline
\end{tabular}

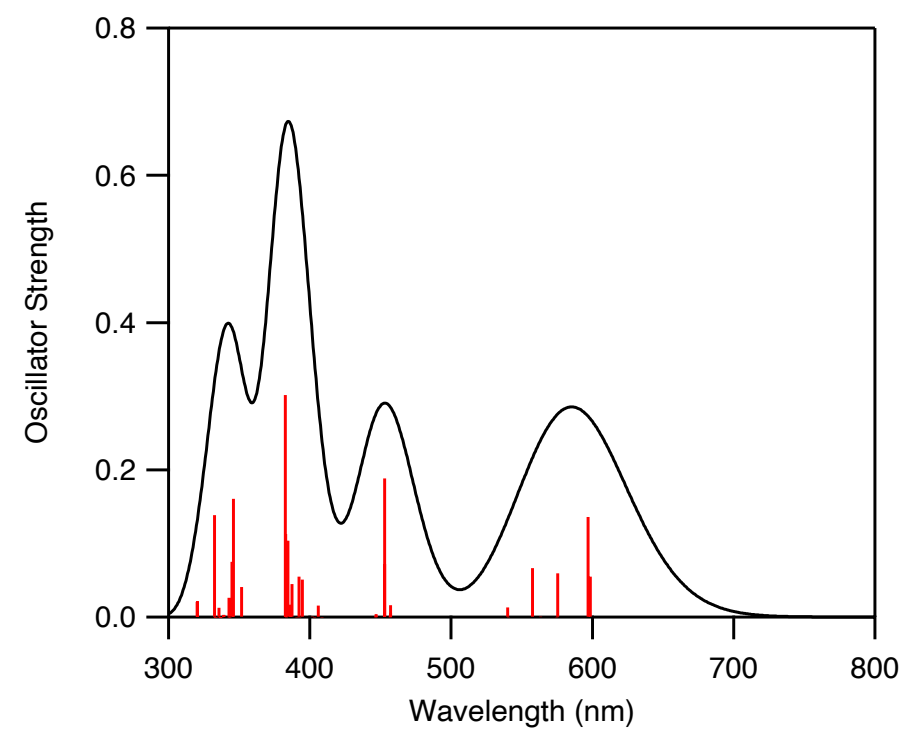

Figure S24. Simulated UV-vis spectrum for 4, from TD-DFT calculations (for clarity, only predicted transitions with an oscillator strength $\geq 0.03$ are shown). 


\section{References}

1. Pangborn, A. B.; Giardello, M. A.; Grubbs, R. H.; Rosen, R. K.; Timmers, F. J. Safe and Convenient Procedure for Solvent Purification. Organometallics 1996, 15, 518.

2. Vu, C.; Walker, D.; Wells, J.; Fox, S. Elaboration of 1,8-naphthyridine-2,7-dicarboxaldehyde into novel 2,7-dimethylimine derivatives. J. Heterocyclic Chem. 2002, 39, 829.

3. Fulmer, G. R.; Miller, A. J. M.; Sherden, N. H.; Gottlieb, H. E.; Nudelman, A.; Stoltz, B. M.; Bercaw, J. E.; Goldberg, K. I. NMR Chemical Shifts of Trace Impurities: Common Laboratory Solvents, Organics, and Gases in Deuterated Solvents Relevant to the Organometallic Chemist. Organometallics 2010, 29, 2176.

4. Sarkar, S. K.; Upul Ranaweera, R. A. A.; Merugu, R.; Abdelaziz, N. M.; Robinson, J.; Day, H. A.; Krause, J. A.; Gudmundsdottir, A. D. Comparison of the Photochemistry of Acyclic and Cyclic 4-(4-Methoxy-phenyl)-4-oxo-but-2-enoate Ester Derivatives J. Phys. Chem. A 2020, $124,7346$.

5. Version 1.171.37.35 (2014). Oxford Diffraction /Agilent Technologies UK Ltd, Yarnton, England.

6. Sheldrick, G. M. Integrated space-group and crystal-structure determination. Acta Cryst. 2015, A71, 3.

7. Dolomanov, O. V.; Bourhis, L. J.; Gildea, R. J.; Howard, J. A. K.; Puschmann, H. OLEX2: a complete structure solution, refinement and analysis program. J. Appl. Cryst. 2009, 42, 339.

8. CrystalMaker Software Ltd, Oxford, England (www.crystalmaker.com).

9. Gaussian 16, Revision B.01, M. J. Frisch, G. W. Trucks, H. B. Schlegel, G. E. Scuseria, M. A. Robb, J. R. Cheeseman, G. Scalmani, V. Barone, G. A. Petersson, H. Nakatsuji, X. Li, M. Caricato, A. V. Marenich, J. Bloino, B. G. Janesko, R. Gomperts, B. Mennucci, H. P. Hratchian, J. V. Ortiz, A. F. Izmaylov, J. L. Sonnenberg, D. Williams-Young, F. Ding, F. Lipparini, F. Egidi, J. Goings, B. Peng, A. Petrone, T. Henderson, D. Ranasinghe, V. G. Zakrzewski, J. Gao, N. Rega, G. Zheng, W. Liang, M. Hada, M. Ehara, K. Toyota, R. Fukuda, J. Hasegawa, M. Ishida, T. Nakajima, Y. Honda, O. Kitao, H. Nakai, T. Vreven, K. Throssell, J. A. Montgomery, Jr., J. E. Peralta, F. Ogliaro, M. J. Bearpark, J. J. Heyd, E. N. Brothers, K. N. Kudin, V. N. Staroverov, T. A. Keith, R. Kobayashi, J. Normand, K. Raghavachari, A. P. Rendell, J. C. Burant, S. S. lyengar, J. Tomasi, M. Cossi, J. M. Millam, M. Klene, C. Adamo, R. Cammi, J. W. Ochterski, R. L. Martin, K. Morokuma, O. Farkas, J. B. Foresman, and D. J. Fox, Gaussian, Inc., Wallingford CT, 2016.

10. GaussView, Version 6, Roy Dennington, Todd A. Keith, and John M. Millam, Semichem Inc., Shawnee Mission, KS, 2016.

11. Chemissian version 4.60, ( Skripnikov Leonid 2005-2018, www.chemissian.com 